\title{
Time-Frequency Feature Extraction Method of the Multi-Source Shock Signal Based on Improved VMD and Bilateral Adaptive Laplace Wavelet
}

\section{Nanyang Zhao}

Beijing University of Chemical Technology

Jinjie Zhang ( $\square$ zjj87427@163.com )

Beijing University of Chemical Technology https://orcid.org/0000-0001-9516-0127

Zhiwei Mao

Beijing University of Chemical Technology

Zhinong Jiang

Beijing University of Chemical Technology

He Li

Beijing University of Chemical Technology

Original Article

Keywords: Shock, Signal processing, Wavelet, VMD, Fault diagnosis, Diesel engine

Posted Date: January 4th, 2021

DOI: https://doi.org/10.21203/rs.3.rs-134820/v1

License: (c) (i) This work is licensed under a Creative Commons Attribution 4.0 International License.

Read Full License 


\section{Title page}

\section{Time-Frequency Feature Extraction Method of the Multi-Source Shock Signal Based on Improved VMD and Bilateral Adaptive Laplace Wavelet}

Nan-Yang Zhao received the B.S. degree from Beijing University of Chemical Technology, Beijing, China, in 2018, where he is currently pursuing for the Ph.D. His current research interest includes intelligent fault diagnosis of reciprocating machinery, mainly for diesel engines and reciprocating compressors.

E-mail: zhaony1995@163.com

Jin-Jie Zhang received a Ph.D. degree from Beijing University of Chemical Technology, Beijing, China, in 2014. He stayed in the university after his postdoctoral study in 2016. Engaged in research on online monitoring and intelligent diagnosis technology of reciprocating machinery faults, he has published more than 20 papers in the field of fault monitoring and diagnosis of reciprocating compressors, won 3 provincial and ministerial level scientific and technological progress awards, and 9 authorized invention patents.

E-mail: zjj87427@163.com

Zhi-Wei Mao received the B.S. and Ph.D. degrees in mechanical engineering from the Beijing University of Chemical Technology, Beijing, China, in 2013 and 2018, respectively. He is currently a postdoctoral research fellow with Beijing University of Chemical Technology. His research interests include deep learning and transfer learning, and their application in condition monitoring, intelligent diagnosis and predictive maintenance.

E-mail: maozhiwei@mail.buct.edu.cn

Zhi-Nong Jiang, professor and doctoral supervisor. Deputy Director of the Research Center for Diagnosis and Self-Healing Engineering, Beijing University of Chemical Technology, Executive Director of the Chinese Society of Vibration Engineering, Executive Director of the Fault Diagnosis Committee of the Chinese Society of Vibration Engineering. He has published over 100 academic papers in the past five years. He won 3 provincial and ministerial level scientific and technological progress awards and 18 authorized invention patents. He has undertaken more than 10 vertical projects including National 973, National 863, National Key R\&D Program, National Defense 973, and National Defense Research, and has undertaken more than 10 enterprise projects such as petroleum and petrochemical. Presided over the research and development of the first online monitoring and diagnosis system for reciprocating compressor units in China and achieved widespread promotion and application.

E-mail: jiangzhinong@263.net

He Li received the B.S. degree from Beijing University of Chemical Technology, Beijing, China, in 2019, where he is currently pursuing for the Ph.D. His current research interest includes fault diagnosis of diesel engines and signal processing.

E-mail: 15010701296@163.com

Corresponding author: Jin-Jie Zhang E-mail: zjj87427@163.com; Zhi-Wei Mao Email: maozhiwei@mail.buct.edu.cn 


\title{
Time-Frequency Feature Extraction Method of the Multi-Source Shock Signal Based on Improved VMD and Bilateral Adaptive Laplace Wavelet
}

\author{
Nan-Yang Zhao •Jin-Jie Zhang $\bullet$ Zhi-Wei Mao $・$ Zhi-Nong Jiang $\bullet \mathrm{He} \mathrm{Li}$
}

(C) Chinese Mechanical Engineering Society and Springer-Verlag Berlin Heidelberg 2020

\begin{abstract}
Reciprocating machinery, e.g., diesel engines and reciprocating compressors, is the key power component in petroleum, petrochemical, nuclear power, and shipbuilding industries. Vibration signals have the characteristics of multisource strong shock coupling and strong noise interference owing to the complex structure of reciprocating machinery; therefore, it is difficult to extract, analyze, and diagnose mechanical fault features. Moreover, failures occur frequently every year, causing serious economic losses. To accurately and efficiently extract sensitive features from the strong noise interference and unsteady monitoring signals of reciprocating machinery, a study on the timefrequency feature extraction method of multi-source shock signals was conducted. Combining the characteristics of reciprocating mechanical vibration signals, a targeted optimization method considering the variational modal decomposition (VMD) mode number $\mathrm{K}$ and second penalty factor $\alpha$ was proposed, which completed the adaptive decomposition of coupled signals. Aiming at the bilateral asymmetric attenuation characteristics of reciprocating mechanical shock signals, a new bilateral adaptive Laplace wavelet (BALW) was established. A search strategy for wavelet local parameters of multi-impact signals was proposed using the harmony search (HS) method. A multi-source shock simulation signal was established and actual data of the valve fault were obtained through diesel engine fault experiments. The test results demonstrated that the new method achieved adaptive extraction of local shock features of non-stationary multi-source shock signals and was superior to the original method in terms of signal decomposition effect, sensitive feature extraction, fault recognition accuracy, and parameter search time. The fault recognition rate of the intake and exhaust valve clearance was above $90 \%$ and the extraction accuracy of the shock start position was improved by $10^{\circ}$.
\end{abstract}

Keywords: Shock $\cdot$ Signal processing $\bullet$ Wavelet $・$ VMD $\bullet$ Fault diagnosis $\bullet$ Diesel engine

Jin-Jie Zhang; Zhi-Wei Mao

zjj87427@163.com; maozhiwei@mail.buct.edu.cn

\section{Introduction}

High-end mechanical equipment, such as high-power gas turbines, diesel engines, high-pressure reciprocating compressors, and large centrifugal pumps, are the core power equipment of nuclear power, petrochemicals, shipbuilding, and other industries. The development of fault monitoring, diagnosis, and health management technology for key equipment has always attracted the attention of researchers [1]-[2]. The feature extraction of equipment operating parameters was the basis for establishing effective fault diagnosis and state evaluation models [3]-[4]. Accurate and efficient extraction of sensitive features from unsteadystate monitoring signals with a lot of noise interference is a difficult problem to address owing to the practical effects of early fault warning and diagnosis methods [5].

According to the working principle and failure mechanism of different types of equipment, research has focused on fault feature extraction methods for specific equipment. Rotating machinery, e.g., rotors, bearings, and gears, has a close correlation with characteristic frequencies, such as rotation frequency, passing frequency, and meshing frequency, in their dynamic characteristics under failure. Therefore, the focus of related research was on effectively extracting the frequency domain characteristics of early weak faults from the signals of non-stationary conditions [6]-[8]. The work of reciprocating mechanical parts was periodic. The movement and force change laws of the pistons and connecting rods were directly related to the crankshaft angle and the periodic time domain and timefrequency characteristics of the fault signal were obvious. For example, diesel engine valve wear failure results in changes to the valve opening and closing impact angle and peak value [9]. Similarly, a reciprocating compressor connecting rod small end bearing wear fault results in a

Beijing Key Laboratory of High-end Mechanical Equipment Health Monitoring and Self-Recovery, Beijing University of Chemical Technology, Beijing 100029, China 
crosshead pin force reversal angle shock [10]. However, owing to the non-stationary multi-source strong shock coupling and strong noise interference characteristics of the reciprocating mechanical vibration signals, it is important to identify the shock source from the complex interference signals and extract the time-frequency domain characteristics of the shocks.

Representative methods for decomposition processing and feature extraction of various signals include wavelet transform (WT), empirical mode decomposition (EMD), ensemble empirical mode decomposition (EEMD), variational modal decomposition (VMD), etc. Recently, new methods based on neural networks have been proposed, including autoencoders (AEs) and convolutional neural networks (CNNs).

Research has been conducted on improving these methods, regarding the above-mentioned feature extraction problems. Based on the existing defects of signal decomposition methods in nonlinear signal analysis and the need to set parameters for WT, EEMD, etc., Pan et al. [11] proposed a symplectic geometry mode decomposition (SGMD) method. Y. Li et al. [12] proposed a bandwidthbased method to select the best envelope interpolation method to improve the EMD frequency band aliasing problem. Focusing on the problem of setting the number of decomposition modes and the second penalty factor in the VMD, C. Zhang et al. [13] combined a genetic algorithm and nonlinear programming to adaptively optimize VMD parameters and apply them to bearing fault diagnosis. Regarding the problem of the fault characteristics of rolling bearings being affected by working conditions, Li et al. [14] proposed a knowledge-mapping adversarial domain adaptive method, which was applied to the bearing feature transfer of a CNN. The existing research mainly focused on the optimization of decomposition methods and the selection of parameters, which are mostly applicable to rotating equipment, such as rolling bearings. There have been few studies on multi-source shock recognition and adaptive time-domain feature extraction of reciprocating machinery and related research has mostly focused on extracting the fault frequency features of rotating machinery. There are still research gaps in the field of time-domain shock feature extraction and fault diagnosis of reciprocating machinery faults. Early fault feature extraction and recognition were difficult problems that plagued equipment health management.

This paper proposes a time-frequency domain feature extraction method for multi-source shock signals based on improved VMD and bilateral adaptive Laplace wavelet (BALW) methods. First, regarding the characteristics of noise interference and multi-source shock coupling of reciprocating mechanical vibration signals, the VMD method was improved and the vibration signals were adaptively decomposed to obtain signals of different frequency bands and their frequency domain characteristics. A new BALW was then constructed to extract the shock and its characteristics in the time and angle domains. Studies on VMD have mostly focused on parameter optimization. Zhao et al. [15] used envelope nesting and a multi-objective function to optimize the two VMD parameters and applied them to the fault diagnosis of rolling bearings. The multisource shocks of the reciprocating mechanical vibration signals had certain differences in both the time and frequency domains. In view of this characteristic, this study optimizes the decomposition mode number $\mathrm{K}$ based on the difference between adjacent decomposition modes. Simultaneously, because the shock frequency band required a higher signal-to-noise ratio (SNR) and the noise component required a larger entropy value, this study combined the normalized SNR of the shock frequency band component and the normalized power spectral entropy value of the main noise frequency band to optimize the second penalty factor $\alpha$.

In terms of shock recognition and feature extraction of the rotating machinery, time-domain feature extraction methods of the shock signals are the current research focus. Based on the vibration characteristics of the second-order underdamped system, the researchers used a Laplace wavelet (LW) to filter and extract the characteristics of the shock [16]-[17]. Regarding the unilateral limitation of the LW, to obtain a wavelet filter with linear phase-frequency response characteristics, researchers proposed an antisymmetric real Laplace wavelet (ARLW). Feng et al. [18] proposed a differential evolution (DE) optimization method and an ARLW filter-based method to extract the impulsive features buried in noisy vibration signals. Dong Wang et al. [19] proposed a Bayesian inference method based on the smoothness index, guided to determine the joint posterior probability distributions of ARLW parameters, which was used to identify different bearing faults. However, the multisource vibration response of reciprocating machinery did not completely conform to the unilateral attenuation characteristics [20]-[21]. Moreover, owing to the sensor characteristics, the collected vibration signals had a certain degree of distortion [22]. Furthermore, ARLW had a symmetrical waveform, which was not fully applicable to the asymmetric vibration and shock signals of the reciprocating machinery. Therefore, two different damping ratio parameters were used in this study to establish a new BALW, which better matched the shock vibration shape and 
frequency. In the iterative search of the wavelet parameter interval, the harmony search algorithm [23]-[24] established a BALW parameter search strategy, which solved the automatic search problem of wavelet parameters of different time-domain shock components. Based on the diesel engine test bench, fault simulation and data collection were conducted and the actual application effects of the new method proposed in this paper were verified by the diesel engine vibration signals.

The contributions of the new method demonstrated in this paper can be summarized as follows: (1) Combining the characteristics of reciprocating mechanical signals, a parameter optimization method based on VMD was proposed. The simulation signal and actual diesel engine vibration signal processing results showed that the new method effectively extracted the shock components of each frequency band. (2) The proposed BALW had better matching performance to the actual shock signal and resulted in better accuracy when extracting the time-domain features of the shock signal, such as the shock start position and shock frequency characteristics. (3) The multi-shock signal wavelet parameter search strategy based on the HS algorithm significantly improved the speed and effect. (4) According to the fault data, the frequency and time-domain shock characteristics of the shock signal were extracted, including the shock start position, peak frequency, peak value, root mean square, standard deviation, crest factor, kurtosis, etc., which improved the ability to capture the local features of the early fault impact. The fault recognition accuracy rate exceeded $90 \%$. Moreover, the extraction accuracy of the shock start position was improved by $10^{\circ}$.

This paper is structured as follows. Section 2 introduces the optimized VMD algorithm and the principle of the BALW. Section 3 introduces the application effect of the method proposed in this study on the simulated signal. Section 4 describes the experimental equipment and experimental data application effects. Finally, the conclusions are presented in Section 5 .

\section{Theory and Method}

\subsection{VMD Parameter Optimization Method}

Diesel engine vibration included the shock response of various components and there was aliasing of multi-band signals. The fault-sensitive shock signals must be separated from them. Therefore, this study used the widely used VMD method to decompose diesel engine vibration signals. In the VMD method, it is necessary to set the modal number $\mathrm{K}$ and the second penalty factor $\alpha$ in advance; however, it is difficult to set these two parameters in practice. This paper proposed a VMD parameter optimization method based on the characteristics of large shock vibration signals.

\subsubsection{Optimization of the Second Senalty Factor}

The second penalty factor, $\alpha$, affects the signal reconstruction accuracy; it is a significant advantage that the decomposed shock signal contains less noise interference. The shock components in the vibration signal often exist in the mid-band of the signal so the initial decomposition mode number, $K=3$, is decomposed into three parts: lowfrequency IMF1, intermediate-frequency IMF2, and highfrequency IMF3. The SNR is calculated for the decomposed signal of the intermediate frequency; a higher SNR is desired. The SNR calculation formula is as follows:

$$
S N R=R M S(I M F 2) / R M S\left(I M F 2 \_ \text {noise }\right),
$$

where the RMS is the root mean square value, IMF2 is the decomposed mid-band signal, and IMF2_noise is the decomposed non-shock component of the mid-band.

For the high-frequency part, the main component is noise and the entropy value of the noise component is relatively large; therefore, a high-frequency component, IMF3, with a higher power spectrum entropy value must be obtained. The power spectrum of each VMD component is calculated as follows:

$$
P_{I M F}=\left|F_{I M F}(f)\right|^{2} / N,
$$

where $F_{I M F}(f)$ is the discrete signal Fourier transform of the component and $N$ is the data length.

The power spectrum entropy value is:

$$
H_{P}=-\sum_{i=1}^{N} q_{i} \log q_{i}
$$

where $q_{i}$ is the proportion of the $i$-th power spectrum value in the entire spectrum.

The optimization target of the second penalty factor $\alpha$ is the maximum value of the sum of the SNR of the normalized intermediate frequency component and the power spectrum entropy of the normalized high-frequency component. The optimization objective function is as follows:

$$
\begin{aligned}
F(\alpha)= & \text { noramlization }(\operatorname{SNR}(\alpha)) \\
& + \text { noramlization }\left(H_{P}(\alpha)\right)
\end{aligned}
$$


where noramlization $(\operatorname{SNR}(\alpha))$ is the normalized SNR vector and noramlization $\left(H_{P}(\alpha)\right)$ is the normalized power spectrum entropy vector.

\subsubsection{Optimization of the Decomposition Mode Number}

During the VMD process of the vibration signal, the frequency band of the signal decomposition becomes finer as the value of $\mathrm{K}$ increases and the difference between adjacent frequency bands becomes smaller. To obtain the best signal decomposition effect, the difference between adjacent frequency band signals should be maximized. Therefore, this study is based on the principle of a minimum average Pearson correlation coefficient between adjacent modal components to iteratively optimize the decomposition mode number $K$. The Pearson correlation coefficient calculation formula for each mode is as follows:

$$
\rho(X, Y)=\frac{\sum(X-\bar{X})(Y-\bar{Y})}{\sqrt{\sum(X-\bar{X})^{2}(Y-\bar{Y})^{2}}} .
$$

According to the number of modes, $K$, the average Pearson correlation coefficient $\rho$ is calculated as follows:

$$
\bar{\rho}=\frac{1}{K-1} \sum_{i=1}^{K-1} \rho\left(I M F_{i}, I M F_{i+1}\right)
$$

\subsection{A New BALW}

Different types of shocks had different shock response performances, mainly due to the different mechanisms and materials. For example, the ignition process of a diesel engine is a combustion process in which a single-point or multiple-point flame spreads to the entire area and the vibration signal response has the characteristics of first rising and then decaying. Therefore, the commonly used LW derived from the Laplace inverse transformation of the second-order underdamped system was not completely suitable for the reciprocating mechanical shock signal. In this study, a BALW was constructed to meet the needs of matching various impact signals.

\subsubsection{Principle of the BALW}

The LW is a complex exponential wavelet with unilateral oscillation attenuation, which conforms to the characteristics of unilateral attenuation of actual shock signals. The complex LW is expressed as: $\psi_{\gamma}(t)=\left\{\begin{array}{ll}\exp \left(-\left(\xi / \sqrt{1-\xi^{2}}\right) 2 \pi f t\right) \exp (-j 2 \pi f t), t \geq 0 \\ 0,\end{array}\right.$,

where the parameter vector $\gamma=\{\xi, f\}$ determines the characteristics of the wavelet. $f \in R^{+}$is the frequency, which determines the LW oscillation frequency. $\xi \in[0,1)$ is the damping ratio, which determines the decay rate of the LW.

The anti-symmetric real LW is treated as antisymmetric on the basis of the LW:

$$
\psi_{\gamma}(t)=\exp \left(-\left(\xi / \sqrt{1-\xi^{2}}\right) 2 \pi f|t|\right) \exp (-j 2 \pi f t)
$$

The BALW is proposed on the basis of the ARLW, which has the characteristics of bilateral asymmetric attenuation. When the unilateral damping value is large, the waveform characteristics are close to the unilateral damping type impact. When the bilateral damping values are both within a reasonable range, the waveform characteristics conform to the impact characteristics of first increasing and then attenuating. Therefore, the BALW has a better impact matching ability. The BALW is expressed as:

$$
\psi_{\gamma}(t)=\left\{\begin{array}{l}
\exp \left(-\left(\xi_{1} / \sqrt{1-\xi_{1}^{2}}\right) 2 \pi f t\right) \exp (-j 2 \pi f t), t \geq 0 \\
\exp \left(\left(\xi_{2} / \sqrt{1-\xi_{2}^{2}}\right) 2 \pi f t\right) \exp (-j 2 \pi f t), t<0
\end{array}\right.
$$

where $\xi_{1}, \xi_{2} \in[0,1)$ is the damping ratio.

In the actual signal processing, the real part of the BALW is used, $\operatorname{Re}\left(\psi_{\gamma}\right)$ :

$$
\operatorname{Re}\left[\psi_{\gamma}(t)\right]=\left\{\begin{array}{l}
\exp \left(-\left(\xi_{1} / \sqrt{1-\xi_{1}^{2}}\right) 2 \pi f t\right) \sin (2 \pi f t), t \geq 0 \\
\exp \left(\left(\xi_{2} / \sqrt{1-\xi_{2}^{2}}\right) 2 \pi f t\right) \sin (2 \pi f t), t<0
\end{array}\right.
$$

\subsubsection{Wavelet Parameter Search Method}

The wavelet parameter set is $\phi=F \times Z_{1} \times Z_{2} \times T$, a fourdimensional matrix, and hence the step-by-step iterative search process for each parameter has a significant computational cost; thus, a search algorithm is needed to quickly search for parameters.

The harmony search (HS) algorithm [23] is an 
optimization algorithm inspired by the beautiful harmonizing of musical instruments. It has been widely studied and applied in the field of combinatorial optimization. The main features of the algorithm are fewer setting parameters, strong solution ability of continuous optimization and discrete optimization methods, and a simple concept. This study used the HS algorithm to accelerate the search process of wavelet parameters.

The search objective function is defined as $f(x)$, where $X=\left[F, Z_{1}, Z_{2}, T\right]$. Set the initialized harmony memory bank HM, the HM considering rate (HMCR), the pitch adjusting rate (PAR), the fine-tuning amplitude $b_{w}$, and the maximum number of iterations MAX. HM and HMCR are used to generate a new solution $X_{i}^{\text {new }}$ as follows:

$$
\begin{aligned}
& X_{i}^{\text {new }}=\left[X_{i, 1}^{\text {new }}, \ldots, X_{i, j}^{\text {new }}, \ldots, X_{i, d}^{\text {new }}\right] \\
& X_{i, j}^{\text {new }}=\left\{\begin{array}{ll}
X_{i, j}^{H M} \text { for } \text { rand }<H M C R \\
X_{i, j}^{\text {rand }} \text { for else }
\end{array},\right. \\
& X_{i}^{H M}=\left[X_{i, 1}^{H M}, \ldots, X_{i, j}^{H M}, \ldots, X_{i, d}^{H M}\right] \\
& X_{i}^{\text {rand }}=\left[X_{i, 1}^{\text {rand }}, \ldots, X_{i, j}^{\text {rand }}, \ldots, X_{i, d}^{\text {rand }}\right]
\end{aligned}
$$

$X_{i, j}^{\text {rand }} \quad$ which is a random individual generated in the possible range of values.

The PAR parameter is used to adjust the pitch, as follows:

$$
X_{i}^{\text {new }}=\left\{\begin{array}{l}
X_{i, j}^{H M} \pm \text { rand } \times b_{w} \text { for } \text { rand }<P A R \\
X_{i, j}^{\text {rand }} \text { for else }
\end{array},\right.
$$

The maximum iteration number was selected as the stopping criterion of the analyses in this study, although there are various alternative criterions.

\section{Simulation Signal Processing and Analysis}

\subsection{Simulation Signal Generation}

In actual vibration signals, strong shocks can excite twoorder or even multi-order vibration modes and there is coupling and superposition of different shock signals; hence, single-frequency wavelet matching is not suitable. To reflect the applicability of the multi-impact signal search strategy proposed in this study, a simulation signal $s$ was constructed for verification, where $s_{1}$ was a bilaterally attenuated impulse signal, $s_{2}$ was the double frequency signal of $s_{1}$ at the same time as $\tau_{1}, s_{3}$ was another unilateral attenuation impact signal at time $\tau_{2}$, and $s_{4}$ was the low- frequency sinusoidal signal. The waveform diagram and frequency domain diagram of the simulated signal $s$ are shown in Figures 1 and 2, respectively.

$$
s_{1}=\left\{\begin{array}{l}
A_{1} \exp \left(-\left(\xi_{1} / \sqrt{1-\xi_{1}^{2}}\right) 2 \pi f_{1}\left(t-\tau_{1}\right)\right) \sin \left(2 \pi f_{1}\left(t-\tau_{1}\right)\right), t \geq \tau_{1}, \\
A_{1} \exp \left(\left(\xi_{2} / \sqrt{1-\xi_{2}^{2}}\right) 2 \pi f_{1}\left(t-\tau_{1}\right)\right) \sin \left(2 \pi f_{1}\left(t-\tau_{1}\right)\right), 0 \leq t<\tau_{1}
\end{array},\right.
$$

$s_{2}=\left\{\begin{array}{l}A_{1} \exp \left(-\left(\xi_{1} / \sqrt{1-\xi_{1}^{2}}\right) 4 \pi f_{1}\left(t-\tau_{1}\right)\right) \sin \left(4 \pi f_{1}\left(t-\tau_{1}\right)\right), t \geq \tau_{1} \\ A_{1} \exp \left(\left(\xi_{2} / \sqrt{1-\xi_{2}^{2}}\right) 4 \pi f_{1}\left(t-\tau_{1}\right)\right) \sin \left(4 \pi f_{1}\left(t-\tau_{1}\right)\right), 0 \leq t<\tau_{1}\end{array}\right.$

$s_{3}= \begin{cases}A_{2} \exp \left(-\left(\xi_{3} / \sqrt{1-\xi_{3}^{2}}\right) 2 \pi f_{2}\left(t-\tau_{2}\right)\right) \sin \left(2 \pi f_{2}\left(t-\tau_{2}\right)\right), t \geq \tau_{2} \\ 0, & 0 \leq t<\tau_{2}\end{cases}$

$$
s_{4}=\sin \left(2 \pi f_{3} t\right) \text {, }
$$

$$
s=s_{1}+s_{2}+s_{3}+s_{4}+\text { noise },
$$

where $A_{1}=10, A_{2}=15, \xi_{1}=0.06, \xi_{2}=0.02$, $\xi_{3}=0.04, f_{1}=40 \mathrm{~Hz}, f_{2}=40 \mathrm{~Hz}, f_{3}=10 \mathrm{~Hz}$, $\tau_{1}=3 \mathrm{~s}, \tau_{2}=1 \mathrm{~s}, t \in[0,5] \mathrm{s}$, the noise was Gaussian, and the sampling frequency $F S=400 \mathrm{~Hz}$.

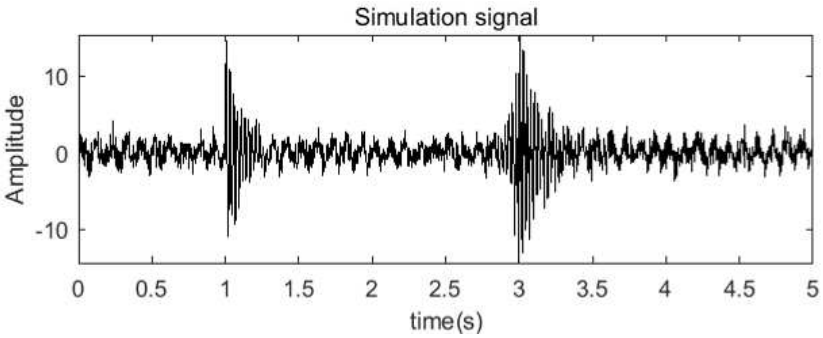

Figure 1 Simulation signal

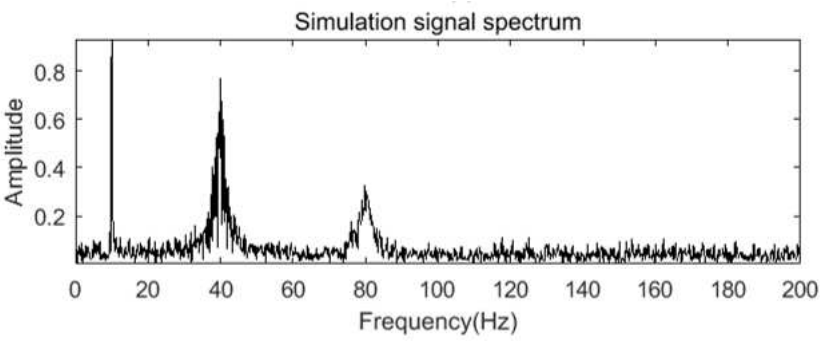

Figure 2 Simulation signal spectrum

\subsection{VMD Processing of the Simulated Signal}

VMD is then performed on the simulated signal. According to the VMD parameter optimization method proposed in this 
paper, the searched optimal secondary penalty factor $\alpha=859$ and the optimal decomposition modal number $K=4$, as shown in Figures 3 and 4 . The VMD modal signals and their spectrograms are shown in Figure 5. It can be seen that VMD effectively decomposed the shock signal components, among which IMF1 was a $40 \mathrm{~Hz}$ signal component and IMF2 was an $80 \mathrm{~Hz}$ signal component.

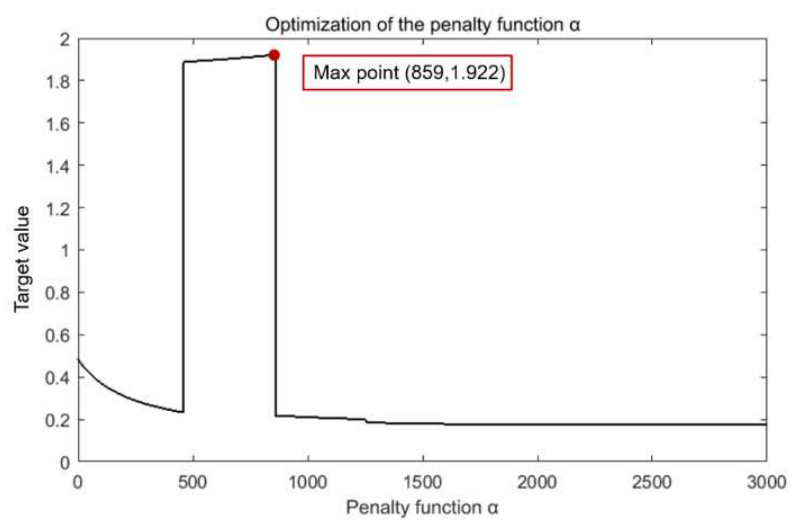

Figure 3 Second penalty factor VMD decomposition iteration

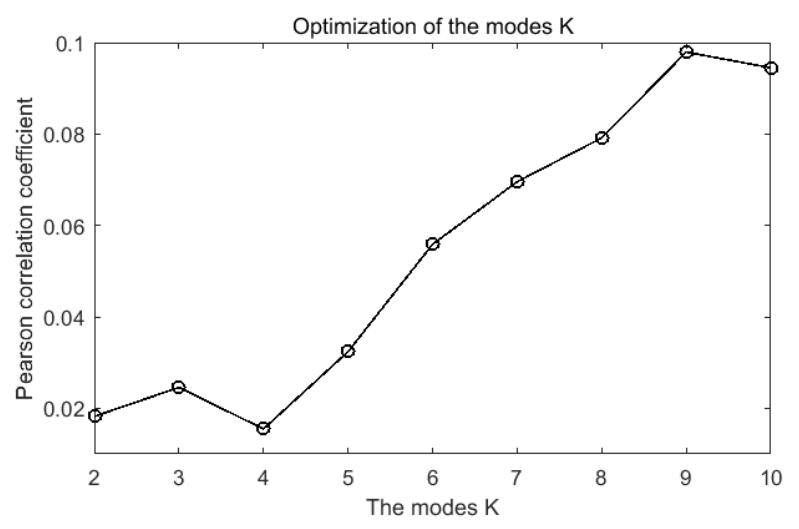

Figure 4 VMD decomposition iteration of the modes $K$
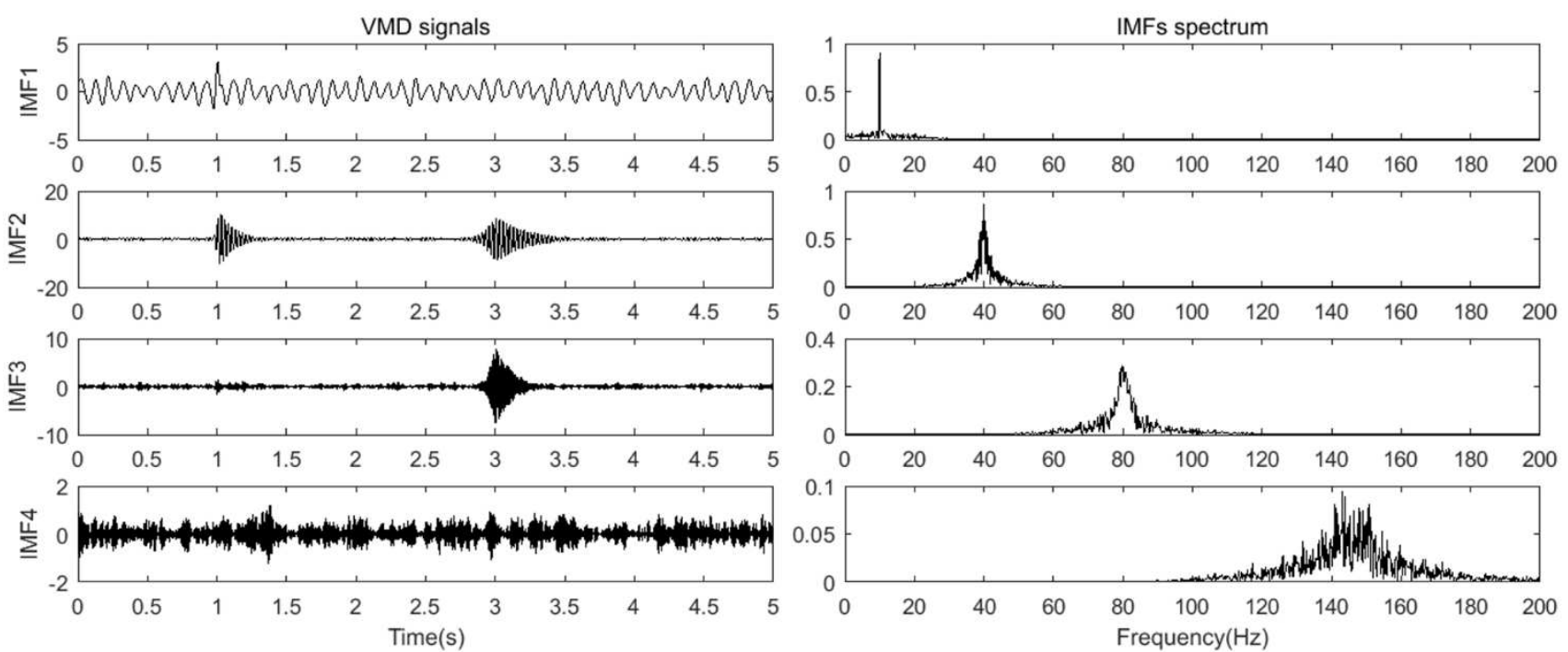

Figure 5 VMD decomposition mode signals and their frequency spectrums

Table 1 The similarity of the impact signal components before and after decomposition

\begin{tabular}{lcc}
\hline & $\alpha=859, K=4$ & $\alpha=2000, K=5$ \\
\hline $\operatorname{Dist}\left(y_{2}, I M F y_{2}\right)$ & 0.0284 & 0.0313 \\
$\operatorname{Dist}\left(y_{3}, I M F y_{3}\right)$ & 0.0081 & 0.0097 \\
\hline
\end{tabular}

Comparing the VMD results with optimized parameters $\alpha=859, K=4$ and conventional parameters $\alpha=2000, K=5$ [25], the method proposed in this paper showed that the method proposed in this paper had a better decomposition effect (as shown in Table 1).

To further verify the decomposition effect and advantages of the improved VMD method proposed in this paper, it was also compared with other popular signal decomposition methods in recent years. EMD is often used in fault diagnosis, especially in bearing vibration signal decomposition and feature extraction [26]-[27]. However, there are end effects in the EMD, which affect the decomposition effect. EEMD introduces white noise to improve the end effect [28], but it has the same modal aliasing problem as EMD. EEMD processing was conducted on the simulation signal and there were problems 
of frequency band aliasing and impact signal decomposition (as shown in Figure 6), which showed the superiority of the
VMD method.
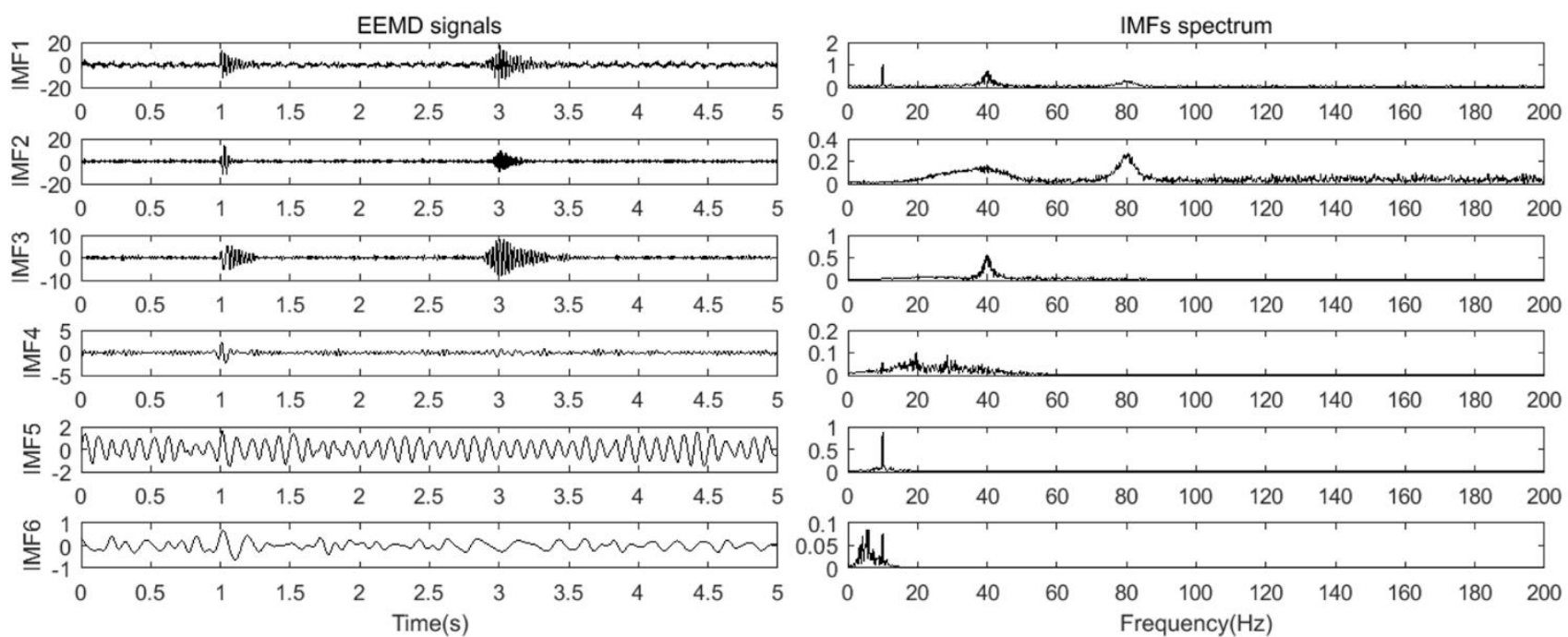

Figure 6 The simulation signal EEMD components and their spectrum

\subsection{Time-Frequency Feature Extraction of the Shock Signals}

\subsubsection{Search strategy for wavelet parameters of the multi- shock signals}

A group of vibration signals often contains multiple shock components. During the global search process for the optimal wavelet parameters, there were multiple shocks corresponding to the optimal local parameter values. However, the harmony search algorithm searched for the global optimal solution and only one of the shock components could be found. Therefore, the search algorithm lacked the ability to search for wavelet parameters for multiple shock vibration signals. Aiming at the above problems, a wavelet parameter harmony search strategy for multi-shock signals was proposed.

(1) Perform VMD processing on the original signal to obtain the frequency band signal of the shock component and determine the search frequency interval of each frequency band signal.

(2) Calculate the peak position of each shock frequency band signal separately to determine the time point parameter $\tau$. The time interval $T \in[\tau-\delta, \tau+\delta]$ can be set to capture the peak position more accurately owing to noise interference.

(3) Calculate the best wavelet parameters based on the cosine distance. Set the wavelet envelope Envelope_s cutoff point threshold to 0.01 (the threshold size can be selected and set as required) and zero the signal between the wavelet cut-off points of the original signal.

$$
\text { Envelope_s }=\left\{\begin{array}{l}
\exp \left(-\left(\xi_{1} / \sqrt{1-\xi_{1}^{2}}\right) 2 \pi f(t-\tau)\right), t \geq \tau \\
\exp \left(\left(\xi_{2} / \sqrt{1-\xi_{2}^{2}}\right) 2 \pi f(t-\tau)\right), 0 \leq t<\tau
\end{array},\right.
$$

(4) Repeat (2) and (3) until the cosine distance is less than the threshold of 0.2 so as to complete the wavelet matching parameter search of all shock components.

\subsubsection{Multi-shock recognition of the simulated signal}

The wavelet parameter search strategy of multi-shock signals was used to match the wavelet parameters of the two frequency band signals of IMF1 and IMF2. First, we determine the frequency search interval $F \in\{35: 1: 45,75: 1: 85\}$ and the damping ratio search interval $Z \in\{0.01: 0.01: 0.99\}$. Second, we determine the $\mathrm{HMS}=20, \quad \mathrm{HMCR}=0.5, \quad \mathrm{PAR}=0.5, \quad b_{w}=5 \quad$ and $\mathrm{MAX}=10000$, and then perform the harmony search. The damping ratio at the rising end of the search waveform of the $s_{2}$ signal differed from the actual value by 0.01 , while the remaining parameters matched; this was caused by the loss of the signal decomposition. The damping ratio of the falling end of the search of the $s_{3}$ signal matched and the damping ratio of the rising end reached 0.21 , which was consistent with the original decomposed signal in terms of 
shape and frequency. As shown in Figure 7-10, in terms of vibration waveforms, the decomposed modal signals and the parameter matching signals had a high degree of similarity, indicating that the method proposed in this paper had a good shock parameter extraction result. The specific matching parameters are listed in Table 2.

We analyzed and compared the search results and search

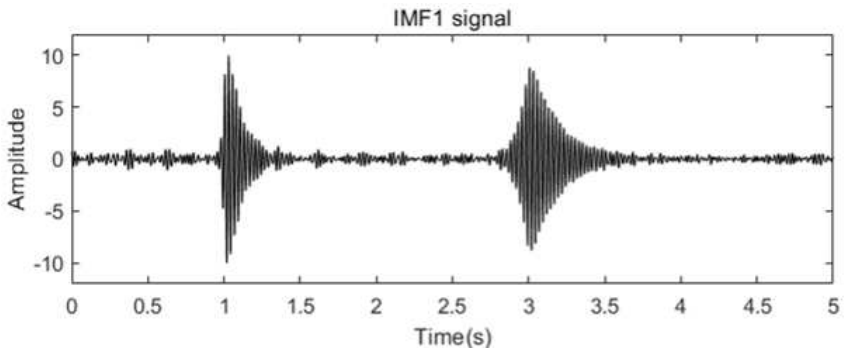

Figure 7 IMF1 signal

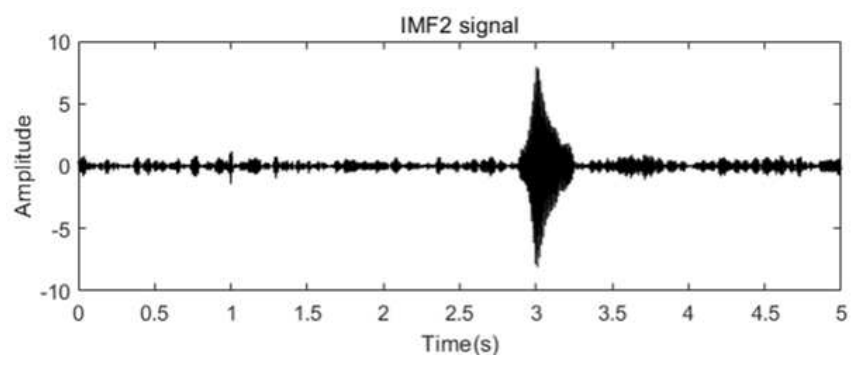

Figure 9 IMF2 signal time of the iterative search, differential evolution algorithm, and harmony search algorithm for the $s_{1}$ signal. All three methods showed consistent search results. The iterative search time was $361.23 \mathrm{~s}$ and the harmony algorithm search time was $3.15 \mathrm{~s}$; these are both better than the differential evolution algorithm search time of $3.53 \mathrm{~s}$.

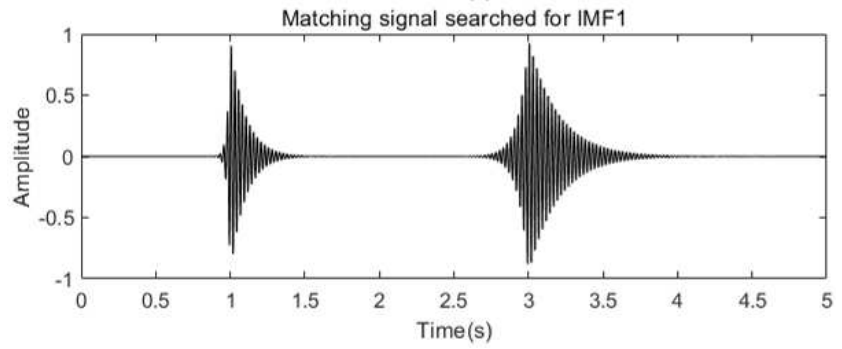

Figure 8 Signals constructed by parameters after searching

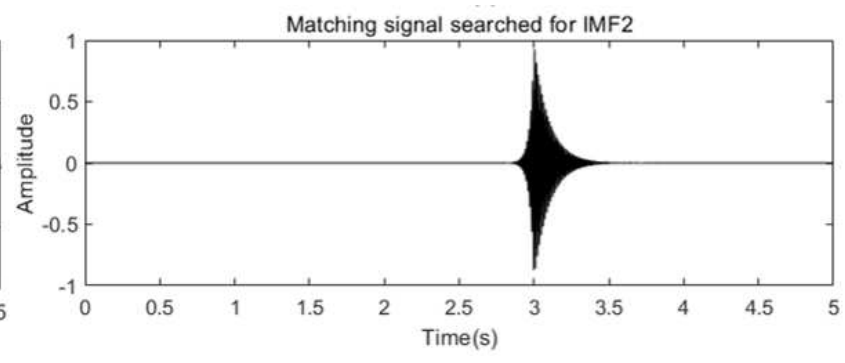

Figure 10 Signals constructed by parameters after searching

Table 2 Comparison of simulation signal search parameters

\begin{tabular}{|c|c|c|c|c|c|c|}
\hline & \multicolumn{2}{|c|}{$s_{1}$} & \multicolumn{2}{|c|}{$s_{2}$} & \multicolumn{2}{|c|}{$s_{3}$} \\
\hline Parameter form & $\begin{array}{c}\text { Actual } \\
\text { parameters }\end{array}$ & $\begin{array}{c}\text { Parameters after } \\
\text { search }\end{array}$ & Actual parameters & $\begin{array}{c}\text { Parameters after } \\
\text { search }\end{array}$ & Actual parameters & $\begin{array}{c}\text { Parameters after } \\
\text { search }\end{array}$ \\
\hline Frequency $(\mathrm{Hz})$ & 40 & 40 & 80 & 80 & 40 & 40 \\
\hline Damping ratio & $0.06 / 0.02$ & $0.06 / 0.02$ & $0.06 / 0.02$ & $0.07 / 0.02$ & $\square / 0.04$ & $0.21 / 0.04$ \\
\hline Time (s) & 3.0 & 3.0 & 3.0 & 3.0 & 1.0 & 1.0 \\
\hline
\end{tabular}

\section{Experimental Equipment and Fault Simulation Data Verification}

\subsection{Test Bench Introduction and Fault Setting}

We conducted a confirmatory experiment on a TBD234V12 direct-injection diesel engine. The common abnormal valve clearance fault of diesel engines was selected as the analysis object. According to the transmission path of vibration, the vibration generated by the valve finally reflects the surface of the diesel engine cylinder head; hence, the upper surface of the diesel engine cylinder head is taken as the vibration measuring point (as shown in Figure 11). This diesel engine is a 12-cylinder diesel engine and its cylinders are divided into columns $\mathrm{A}$ and $\mathrm{B}$. The vibration signals and pulse signals were sampled by the DATA acquisition (DAQ) system. The DAQ card has a 24-bit ADC resolution, with a 


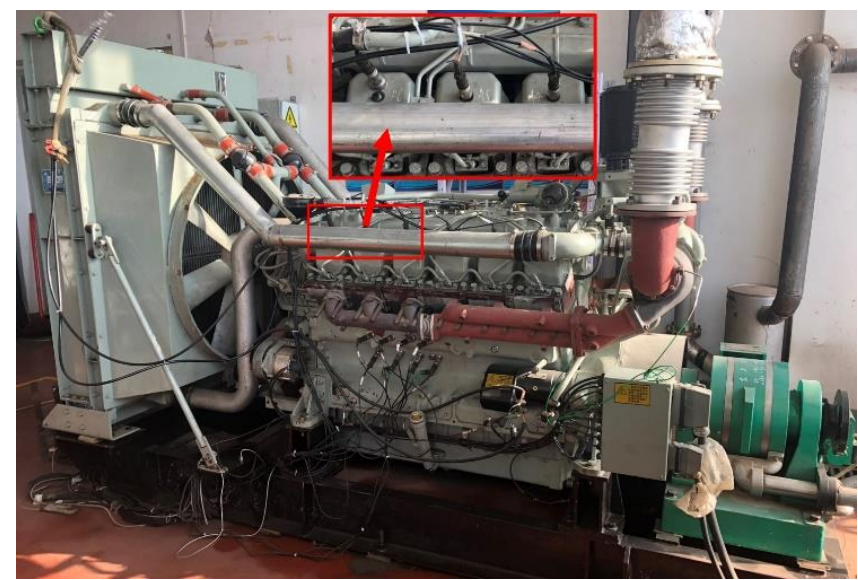

Figure 11 Diesel engine and measuring point installation

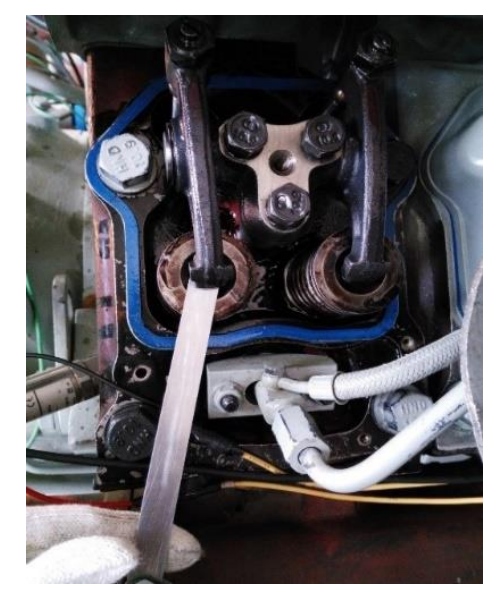

Figure 12 Valve clearance adjustment
Under normal circumstances, the intake valve clearance was $0.3 \mathrm{~mm}$ and the exhaust valve clearance was $0.5 \mathrm{~mm}$. Taking the intake valve as the research object, the B4 cylinder was used in an intake clearance fault simulation experiment. The intake valve clearance was set to $0.3 \mathrm{~mm}$ and $0.9 \mathrm{~mm}$ and the exhaust valve clearance was set to $0.5 \mathrm{~mm}$ and $1.1 \mathrm{~mm}$. When the diesel engine was running at $1500 \mathrm{rpm}, 1800 \mathrm{rpm}$, and $2100 \mathrm{rpm}$, the cylinder head vibration signal was collected.

\subsection{Feature Extraction of Valve Clearance Fault}

According to the flywheel gear pulse signals, the vibration signals were intercepted throughout the period and the timedomain vibration signals were converted to correspond to the crankshaft angle. As shown in Figure 13, when the intake valve clearance of the B4 cylinder is $0.6 \mathrm{~mm}$, the fault signal is compared with the normal signal. The shock amplitude of the intake valve closing increases and the starting position is advanced (the dotted frame area near the $480^{\circ}$ crankshaft angle). However, the valve closing shock amplitude was affected by the operating conditions of the diesel engine and the valve closing shock start phase was relatively fixed; thus the shock start position was a very critical fault feature. From the spectrogram analysis (as shown in Figure 14), the shock frequency band is mainly around $5 \mathrm{kHz}$ but it is difficult to obtain the characteristic frequency of the fault.

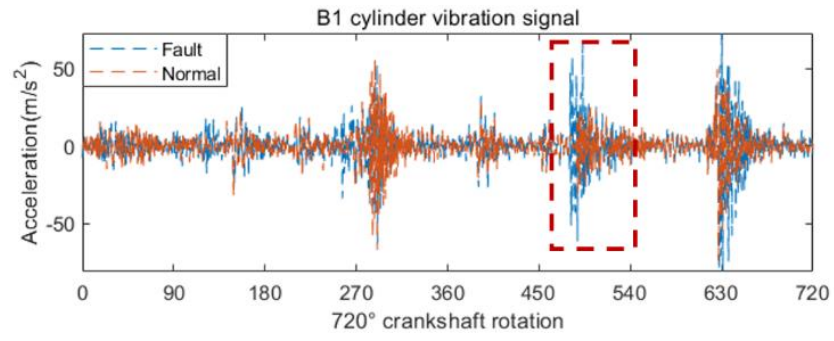

Figure 13 Vibration signal of B4 cylinder under normal and fault conditions

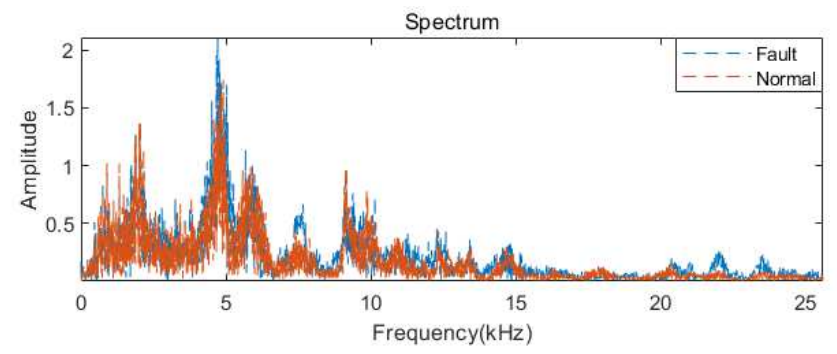

Figure 14 Vibration signal spectrum of B4 cylinder under normal and fault conditions

According to the VMD parameter optimization method proposed in this paper, VMD ( $K=4, \alpha=61$, the iterative process of parameter optimization is shown in Figure 15 and Figure 16) processed modal decomposition signals and their spectra are shown in Figure 17. 


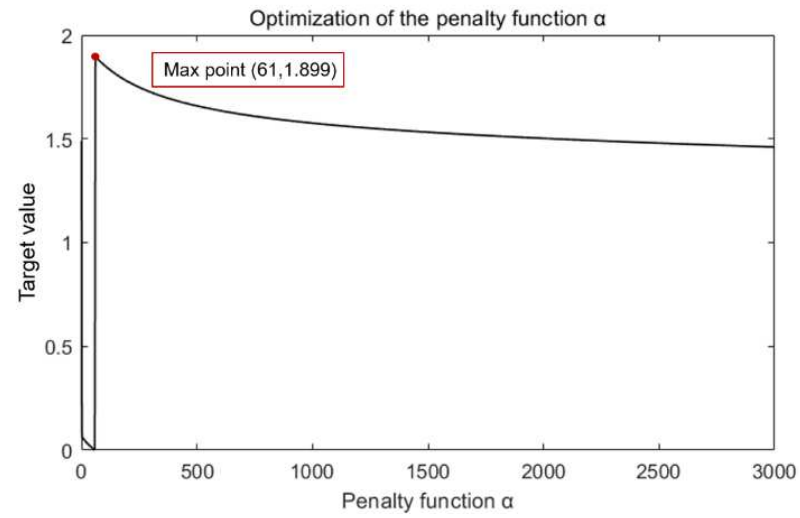

Figure 15 Second penalty factor VMD decomposition iteration
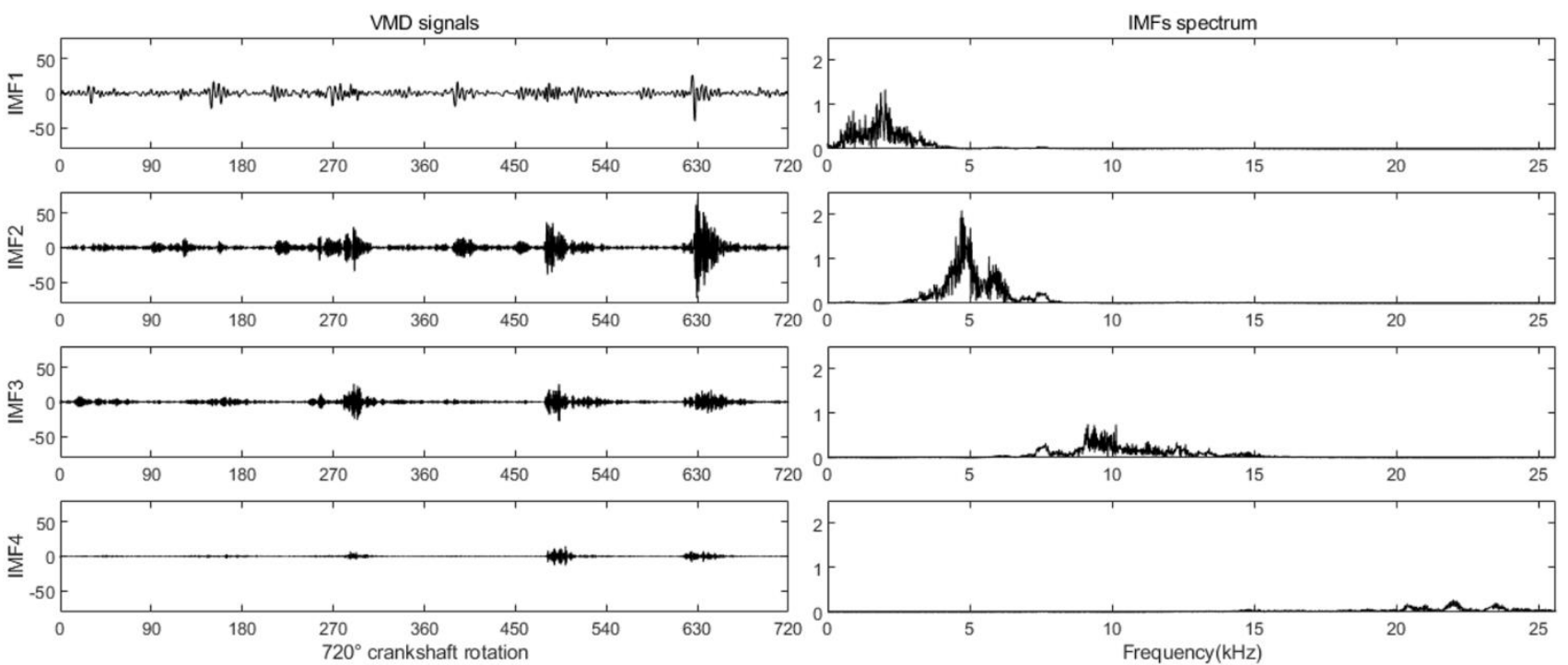

Figure 17 VMD decomposition mode signals and their frequency spectrums

It was determined that the shock frequency band was mainly concentrated at around $5 \mathrm{kHz}$, i.e., the IMF 2 signal. The search frequency band $F \in\{4 \mathrm{k}: 0.1 \mathrm{k}: 6 \mathrm{k}\}$ and the damping ratio range $Z \in\{0.01: 0.01: 0.99\}$ were set, and a wavelet parameter search for the valve closing shock was performed according to the method proposed in this paper. As shown in Figure 18-20, the BALW proposed in this paper has better waveform matching ability for the closing shock of the intake valve after VMD than the LW and ARLW.

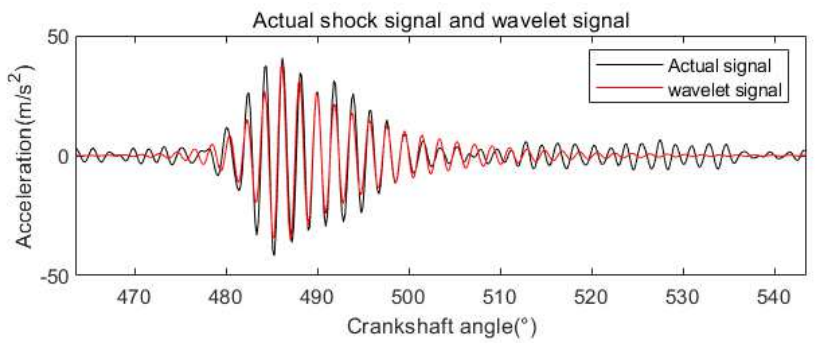

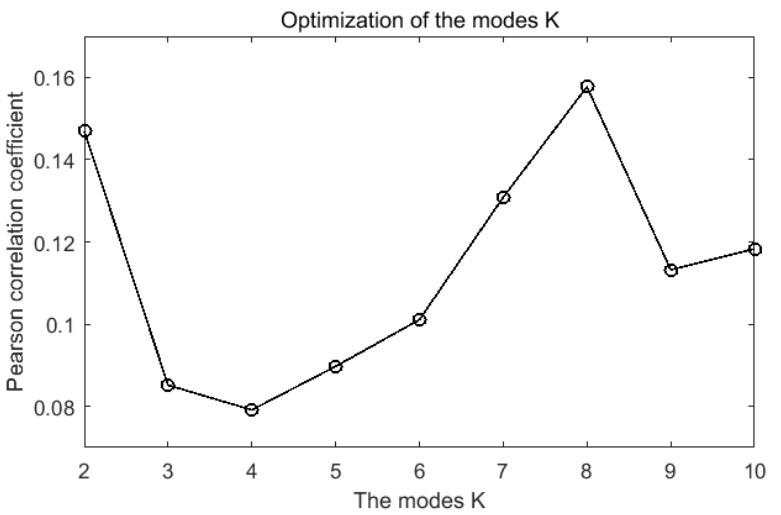

Figure 16 VMD decomposition iteration of the modes $K$ 
Figure 18 BALW search results

Figure 19 LW search results

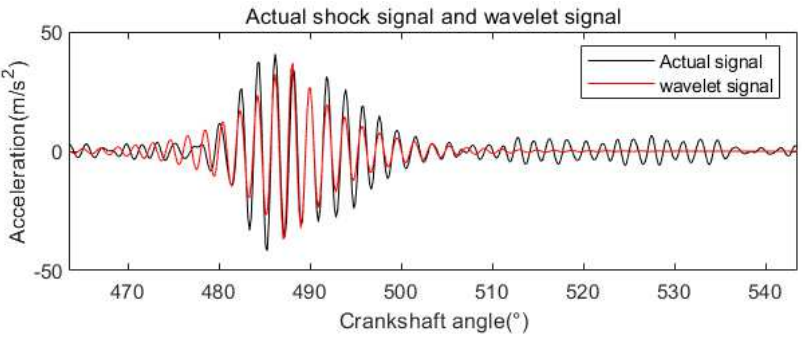

Figure 20 ARLW search results

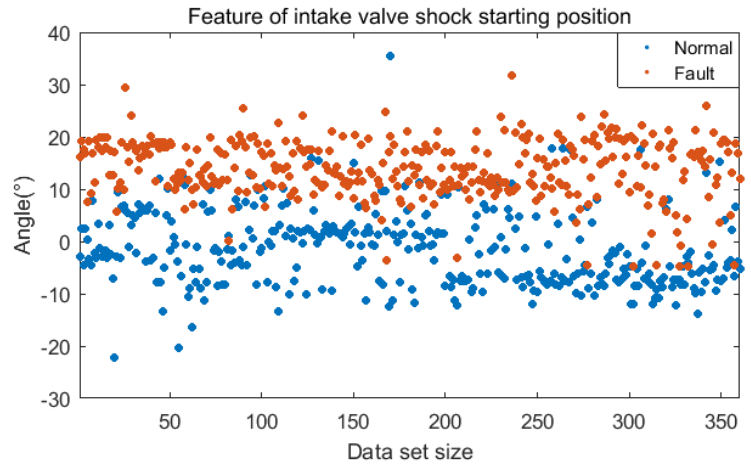

Figure 21 Characteristics of starting position of intake valve closing shock

\subsection{Fault Identification Result}

The time domain, frequency domain, and angle domain features extracted by the method proposed in this paper were used to construct the fault classification feature set. Similarly, the feature set (peak value, RMS, kurtosis, peak frequency, and other common time and frequency domain characteristics) was constructed using the eigenvalues extracted via conventional methods. The commonly used softmax classifier was used for fault classification. There were 240 sets of data for the three speeds, a total of 720 sets, of which normal and fault data each account for half. 480

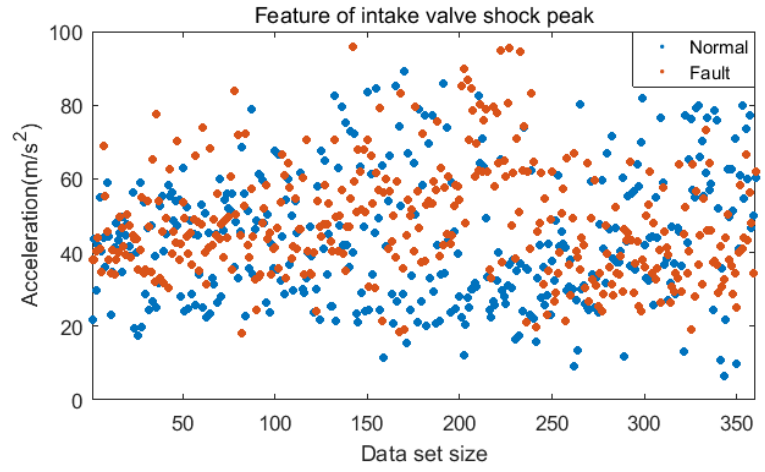

Figure 22 Characteristics of peak of intake valve closing shock

sets of data were randomly selected as the training set and the remaining 360 sets of data as the test set. The clearance fault of the intake and exhaust valve is then recognized.

Table 3 shows the classification results. The fault recognition rate of the intake and exhaust valve clearance of the methods (improved VMD + BALW) proposed in this paper was above $90 \%$ whereas the fault recognition rate of the intake and exhaust valve clearance for the conventional method (VMD + conventional features) was $60-70 \%$. The results showed that the shock feature extraction method proposed in this paper realized the effective extraction of the feature and obtained an accurate fault recognition effect.

Table 3 Comparison of the results of the recognition rate of intake and exhaust valve clearance

Recognition rate of intake and exhaust valve clearance (\%)

\begin{tabular}{ccccc} 
& Normal intake valve & $\begin{array}{c}\text { Abnormal intake } \\
\text { valve }\end{array}$ & $\begin{array}{c}\text { Normal exhaust } \\
\text { valve }\end{array}$ & $\begin{array}{c}\text { Abnormal exhaust } \\
\text { valve }\end{array}$ \\
\hline Improved VMD+BALW & $90.2 \%$ & $90.6 \%$ & $97.4 \%$ & $93.7 \%$ \\
$\begin{array}{c}\text { Improved VMD }+ \\
\text { conventional features }\end{array}$ & $68.5 \%$ & $64.5 \%$ & $67.5 \%$ & $64.0 \%$ \\
\hline
\end{tabular}




\section{Conclusions}

Based on the characteristics of multi-source shock coupling and strong noise interference in the shell vibration signal of reciprocating machinery, this study improved the parameter selection of the VMD method and established a new BALW method for the adaptive extraction of shock features. An optimization strategy based on the harmony search algorithm was proposed for optimizing wavelet parameters. The new method proposed in this paper solved the problem of accurately extracting different types of time-domain shock signals and realized the optimization of wavelet parameters of multi-source shocks and the adaptive extraction of local shock features. A diesel engine test bench was used to obtain abnormal data for simulated valve clearance, the shock feature extraction effect was verified, and the fault recognition accuracy rate exceeded $90 \%$. Moreover, the extraction accuracy of the shock start position was improved by $10^{\circ}$. The main research results of this study are as follows:

(1) Optimize the decomposition mode number $K$ based on the maximum difference between adjacent decomposition modes and optimize the second penalty factor $\alpha$ based on the normalized SNR of the shock frequency components and the normalized power spectrum entropy value of the main noise frequency band. Through comparison of the decomposition effects, the improvement of the VMD method was more suitable for decomposing reciprocating multi-source shock coupling signals and was better than the EMD and traditional VMD methods.

(2) By introducing different bilateral damping ratio parameters to improve the ARLW, a new BALW was established, which had bilateral asymmetric attenuation characteristics. Considering the difficult problem of optimal selection of wavelet local parameters for multishock signals, a wavelet local parameter search strategy for multi-shock signals was established via the harmony search algorithm. The search time was $3.15 \mathrm{~s}$, which improved upon the other methods.

(3) The failure data verification of the diesel engine test bench showed that the new method proposed in this paper realized the adaptive decomposition of the complex multi-impact coupling signals of the diesel engine and local shock feature extraction. Compared with other shock feature extraction methods, it achieved better feature extraction results and fault recognition accuracy.

Aiming at the similarity characteristics of different reciprocating machinery vibration signals, we will conduct further general research on related methods to improve the applicability of the new feature extraction method presented in this paper.

\section{Declaration}

\section{Acknowledgements}

Not applicable

\section{Funding}

Supported by Double First-rate Construction Special Funds (Grant No. ZD1601), National Plan on Key Basic Research and Development (Grant No. 2016YFF0203305).

\section{Availability of data and materials}

The datasets generated and/or analysed during the current study are not publicly available due the high cost of the original data collection, but are available from the corresponding author on reasonable request.

\section{Authors' contributions}

The author' contributions are as follows: NZ and JZ was in charge of the whole trial; NZ and JZ wrote the manuscript; $\mathrm{NZ}, \mathrm{ZM}$ and HL prepared and conducted the experiments. $\mathrm{ZJ}$ provided the materials and funds. All authors read and approved the final manuscript.

\section{Competing interests}

The authors declare no competing financial interests.

\section{Consent for publication}

Not applicable

\section{Ethics approval and consent to participate}

Not applicable

\section{References}

[1] Lei, Y., Yang, B., Jiang, X., Jia, F., Li, N., \& Nandi, A. K. (2020). Applications of machine learning to machine fault diagnosis: A review and roadmap. Mechanical Systems and Signal Processing, 138. doi:10.1016/j.ymssp.2019.106587.

[2] Wang, D., Tsui, K.-L., \& Miao, Q. (2018). Prognostics and Health Management: A Review of Vibration Based Bearing and Gear Health Indicators. Ieee Access, 6. doi:10.1109/access.2017.2774261.

[3] Li, Y., Wang, X., Si, S., \& Huang, S. (2020). Entropy Based Fault Classification Using the Case Western Reserve University Data: A Benchmark Study. Ieee Transactions on Reliability, 69(2), 754-767. doi:10.1109/tr.2019.2896240.

[4] Glowacz, A. (2019). Fault diagnosis of single-phase induction motor based on acoustic signals. Mechanical Systems and Signal Processing, 117, 65-80. doi:10.1016/j.ymssp.2018.07.044. 
[5] Wang, T., Han, Q., Chu, F., \& Feng, Z. (2019). Vibration based condition monitoring and fault diagnosis of wind turbine planetary gearbox: A review. Mechanical Systems and Signal Processing, 126, 662-685. doi:10.1016/j.ymssp.2019.02.051.

[6] Li, D. Z., Wang, W., \& Ismail, F. (2017). An Intelligent Harmonic Synthesis Technique for Air-Gap Eccentricity Fault Diagnosis in Induction Motors. Chinese Journal of Mechanical Engineering, 30(6), 1296-1304. doi:10.1007/s10033-017-0192-3.

[7] Liu, Z., \& Zhang, L. (2020). A review of failure modes, condition monitoring and fault diagnosis methods for large-scale wind turbine bearings. Measurement, 149. doi:10.1016/j.measurement.2019.107002.

[8] Cui, L., Ma, C., Zhang, F., \& Wang, H. (2015). Quantitative Diagnosis of Fault Severity Trend of Rolling Element Bearings. Chinese Journal of Mechanical Engineering, 28(6), 1254-1260. doi:10.3901/cjme.2015.0715.094.

[9] Flett, J., \& Bone, G. M. (2016). Fault detection and diagnosis of diesel engine valve trains. Mechanical Systems and Signal Processing, 72-73, 316-327. doi:10.1016/j.ymssp.2015.10.024.

[10] Jiang, Z., Mao, Z., Yao, Z., Zhang, J., \& Iop. (2015). A Diagnosis method of the small end fault on reciprocating compressor connecting rod. In 9th International Conference on Compressors and Their Systems (Vol. 90).

[11] Pan, H., Yang, Y., Li, X., Zheng, J., \& Cheng, J. (2019). Symplectic geometry mode decomposition and its application to rotating machinery compound fault diagnosis. Mechanical Systems and Signal Processing, 114, 189-211. doi:10.1016/j.ymssp.2018.05.019.

[12] Li, Y., Xu, M., Liang, X., \& Huang, W. (2017). Application of Bandwidth EMD and Adaptive Multiscale Morphology Analysis for Incipient Fault Diagnosis of Rolling Bearings. Ieee Transactions on Industrial Electronics, 64(8), 6506-6517. doi:10.1109/tie.2017.2650873.

[13] Zhang, C., Wang, Y., \& Deng, W. (2020). Fault Diagnosis for Rolling Bearings Using Optimized Variational Mode Decomposition and Resonance Demodulation. Entropy, 22(7). doi:10.3390/e22070739.

[14] Li, Q., Shen, C., Chen, L., \& Zhu, Z. (2021). Knowledge mappingbased adversarial domain adaptation: A novel fault diagnosis method with high generalizability under variable working conditions. Mechanical Systems and Signal Processing, 147. doi:10.1016/j.ymssp.2020.107095.

[15] Zhao, Y., Li, C., Fu, W., Liu, J., Yu, T., \& Chen, H. (2020). A modified variational mode decomposition method based on envelope nesting and multi-criteria evaluation. Journal of Sound and Vibration, 468. doi:10.1016/j.jsv.2019.115099.

[16] Al-Raheem, K. F., Roy, A., Ramachandran, K. P., Harrison, D. K., \& Grainger, S. (2008). Application of the laplace-wavelet combined with ANN for rolling bearing fault diagnosis. Journal of Vibration and Acoustics-Transactions of the Asme, 130(5). doi:10.1115/1.2948399.

[17] Shen, C., Liu, F., Wang, D., Zhang, A., Kong, F., \& Tse, P. W. (2013). A Doppler Transient Model Based on the Laplace Wavelet and Spectrum Correlation Assessment for Locomotive Bearing Fault Diagnosis. Sensors, 13(11), 15726-15746. doi:10.3390/s131115726.

[18] [18] Feng, K., Jiang, Z., He, W., \& Qin, Q. (2011). Rolling element bearing fault detection based on optimal antisymmetric real Laplace wavelet. Measurement, 44(9), 1582-1591. doi:10.1016/j.measurement.2011.06.011.

[19] Wang, D., \& Miao, Q. (2015). Smoothness index-guided Bayesian inference for determining joint posterior probability distributions of anti-symmetric real Laplace wavelet parameters for identification of different bearing faults. Journal of Sound and Vibration, 345, 250266. doi:10.1016/j.jsv.2015.01.052.

[20] Ding, J. (2018). Fault detection of a wheelset bearing in a high-speed train using the shock-response convolutional sparse-coding technique. Measurement, $117, \quad$ 108-124. doi:10.1016/j.measurement.2017.12.010.

[21] Taghizadeh-Alisaraei, A., \& Rezaei-Asl, A. (2016). The effect of added ethanol to diesel fuel on performance, vibration, combustion and knocking of a CI engine. Fuel, 185, 718-733. doi:10.1016/j.fuel.2016.08.041.

[22] Li, L., Liu, G., Zhang, L., \& Li, Q. (2019). Sensor fault detection with generalized likelihood ratio and correlation coefficient for bridge SHM. Journal of Sound and Vibration, 442, 445-458. doi:10.1016/j.jsv.2018.10.062.

[23] Geem, Z. W., Kim, J. H., \& Loganathan, G. V. (2001). A new heuristic optimization algorithm: Harmony search. Simulation, 76(2), 60-68. doi:10.1177/003754970107600201.

[24] Liu, C., Abdulkareem, S. S., Rezvani, A., Samad, S., Aljojo, N., Foong, L. K., \& Nishihara, K. (2020). Stochastic scheduling of a renewable-based microgrid in the presence of electric vehicles using modified harmony search algorithm with control policies. Sustainable Cities and Society, 59. doi:10.1016/j.scs.2020.102183.

[25] Kumar, A., Gandhi, C. P., Zhou, Y., Kumar, R., \& Xiang, J. (2020). Variational mode decomposition based symmetric single valued neutrosophic cross entropy measure for the identification of bearing defects in a centrifugal pump. Applied Acoustics, 165. doi:10.1016/j.apacoust.2020.107294.

[26] [26] Liu, H., Zhang, J., Cheng, Y., \& Lu, C. (2016). Fault diagnosis of gearbox using empirical mode decomposition and multi-fractal detrended cross-correlation analysis. Journal of Sound and Vibration, 385, 350-371. doi:10.1016/j.jsv.2016.09.005.

[27] Chen, D., Lin, J., \& Li, Y. (2018). Modified complementary ensemble empirical mode decomposition and intrinsic mode functions evaluation index for high-speed train gearbox fault diagnosis. Journal of Sound and Vibration, 424, 192-207. doi:10.1016/j.jsv.2018.03.018.

[28] Yang, J., Huang, D., Zhou, D., \& Liu, H. (2020). Optimal IMF selection and unknown fault feature extraction for rolling bearings with different defect modes. Measurement, 157. doi:10.1016/j.measurement.2020.107660. 
Figures

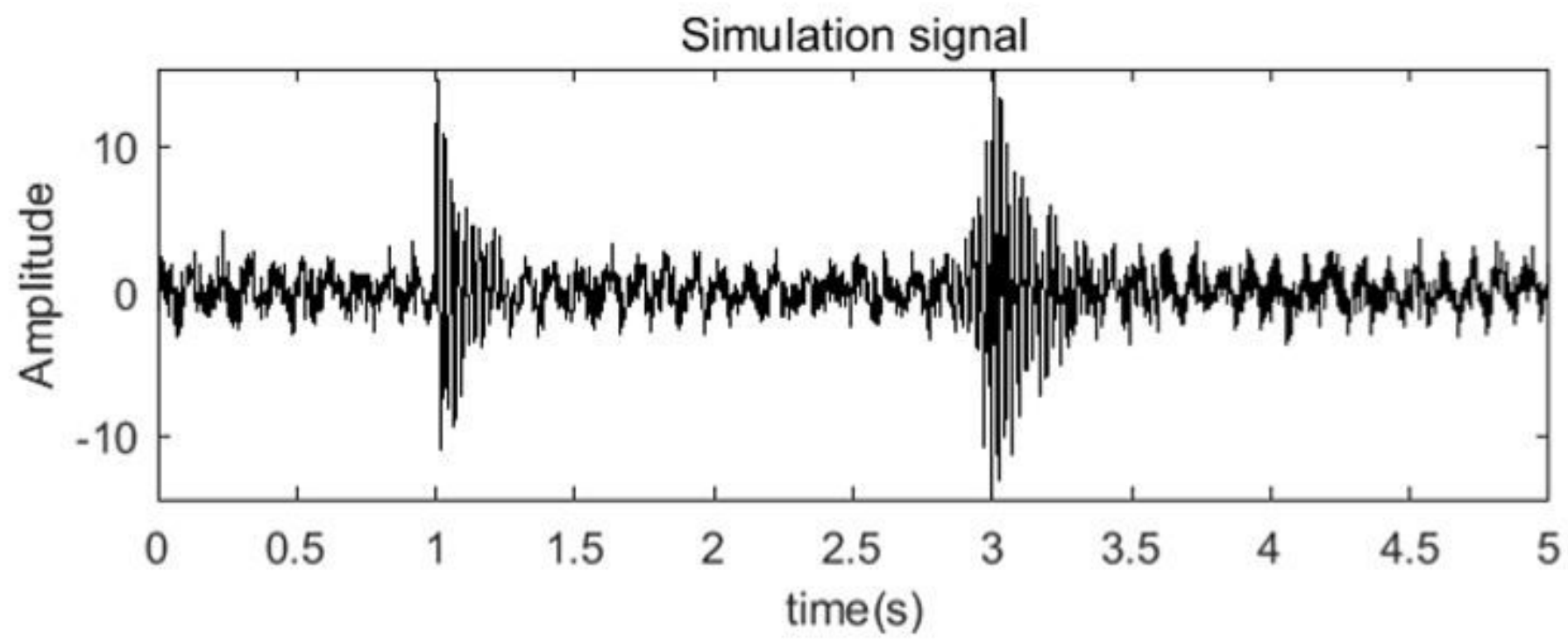

Figure 1

Simulation signal

Simulation signal spectrum

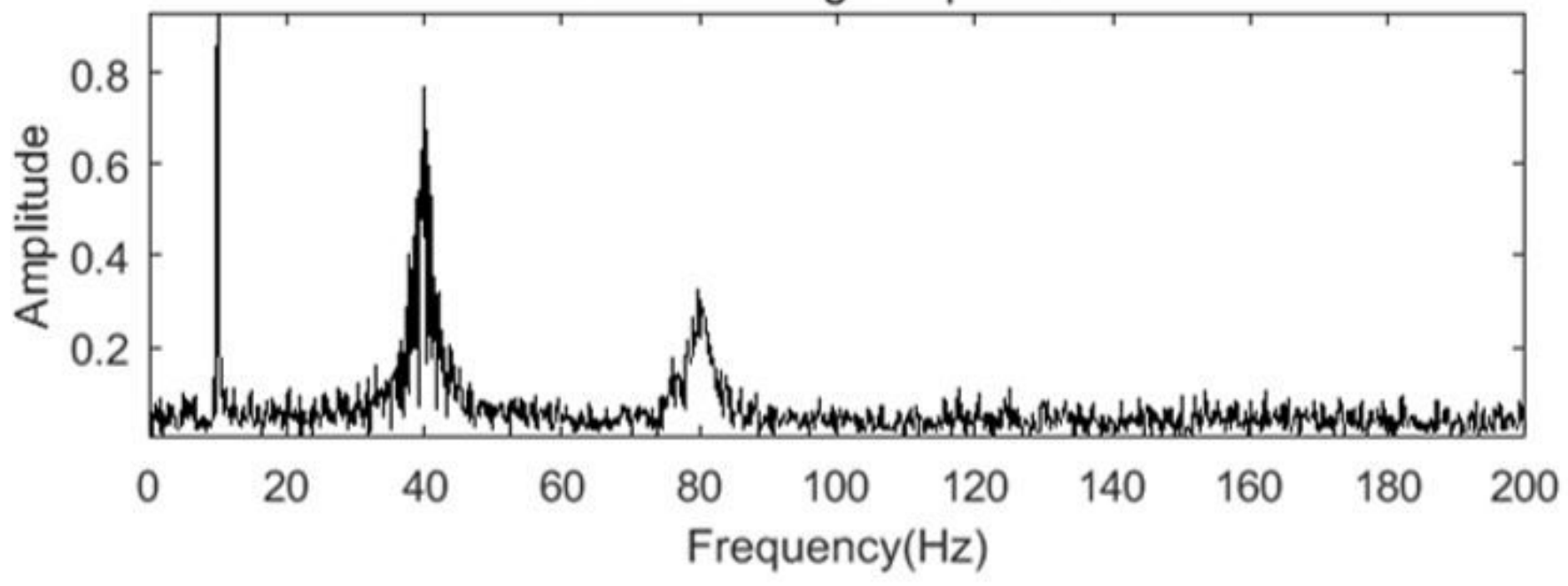

Figure 2

Simulation signal spectrum 


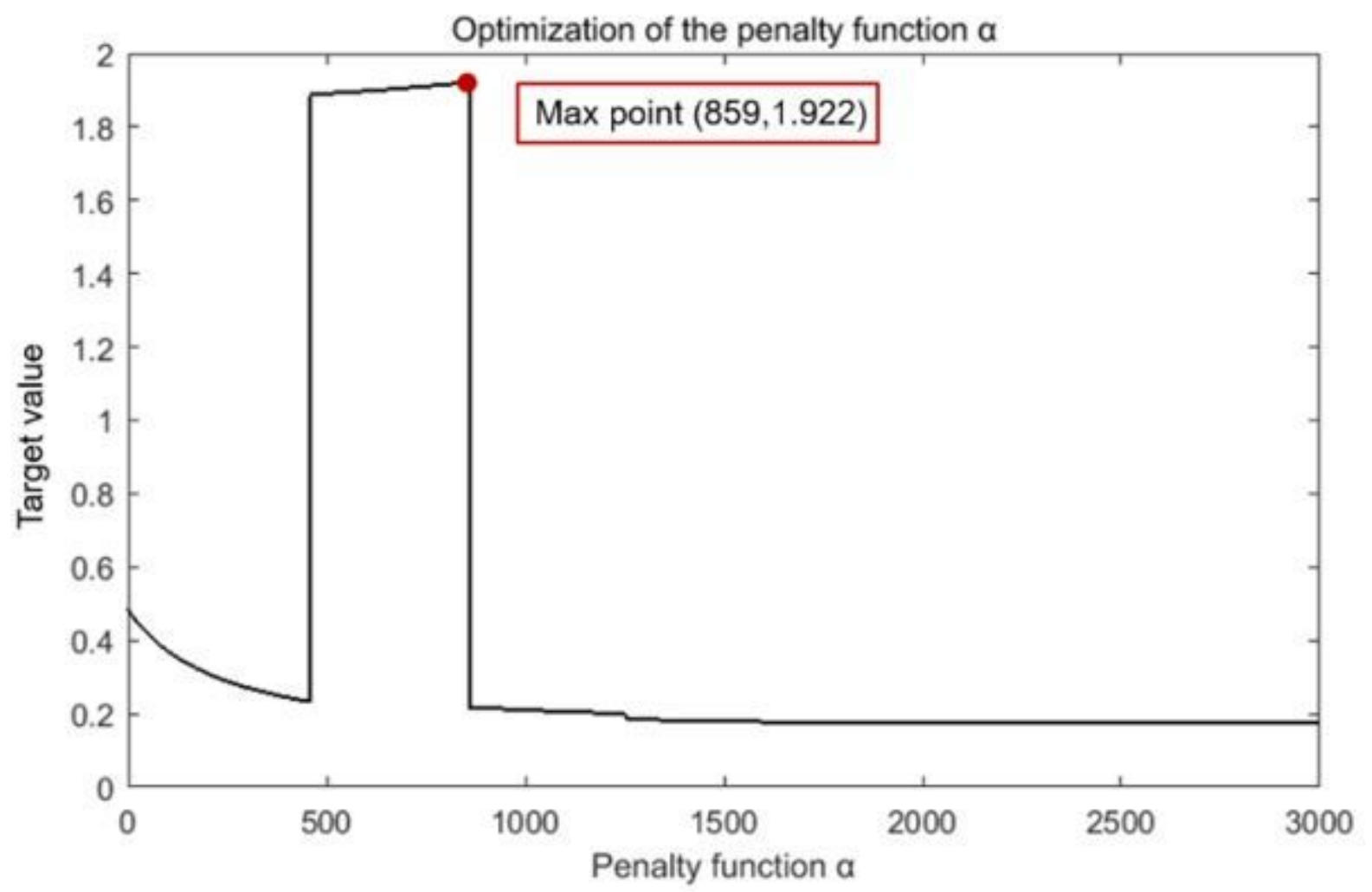

Figure 3

Second penalty factor VMD decomposition iteration 


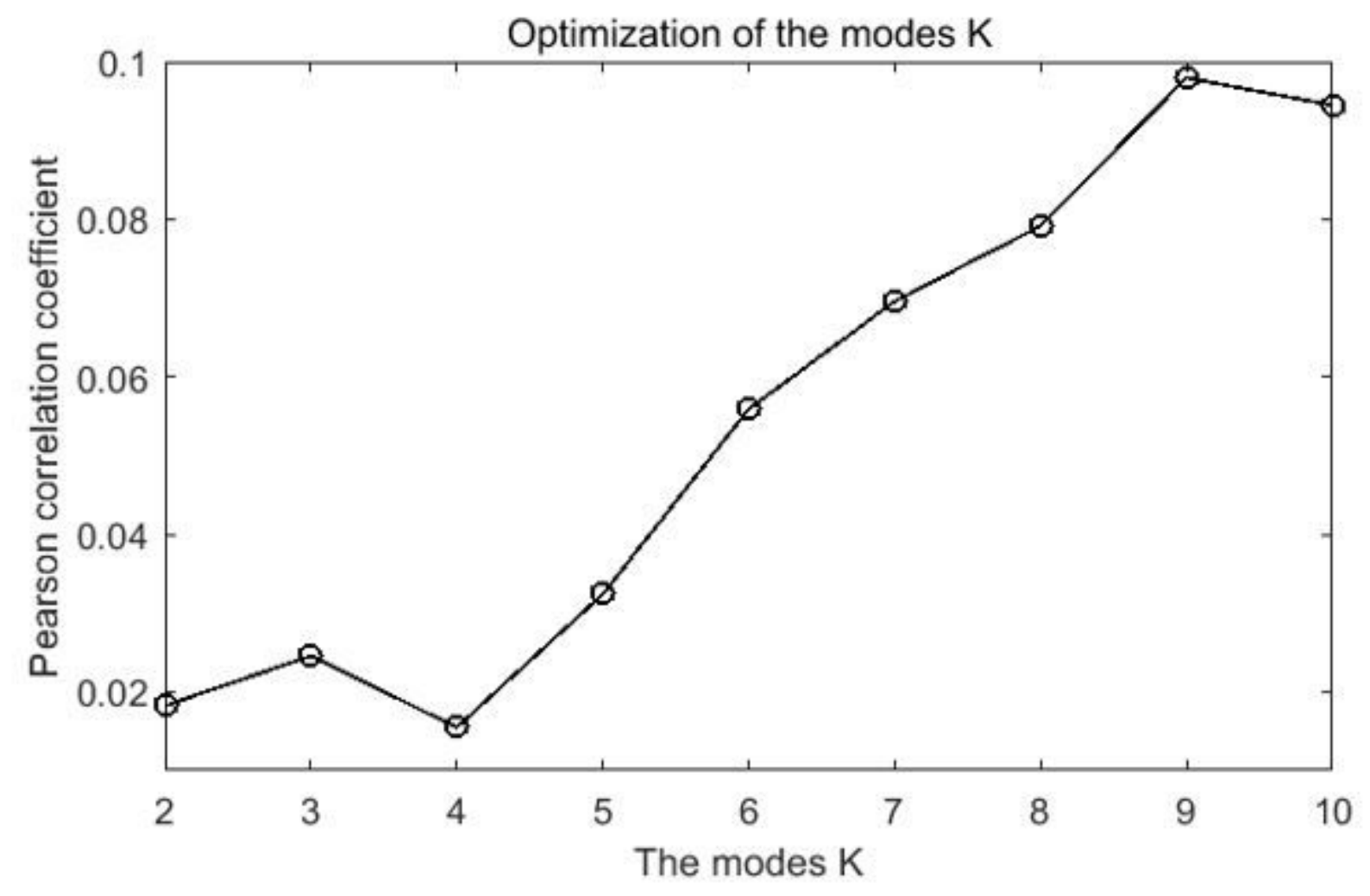

Figure 4

VMD decomposition iteration of the modes $\mathrm{K}$
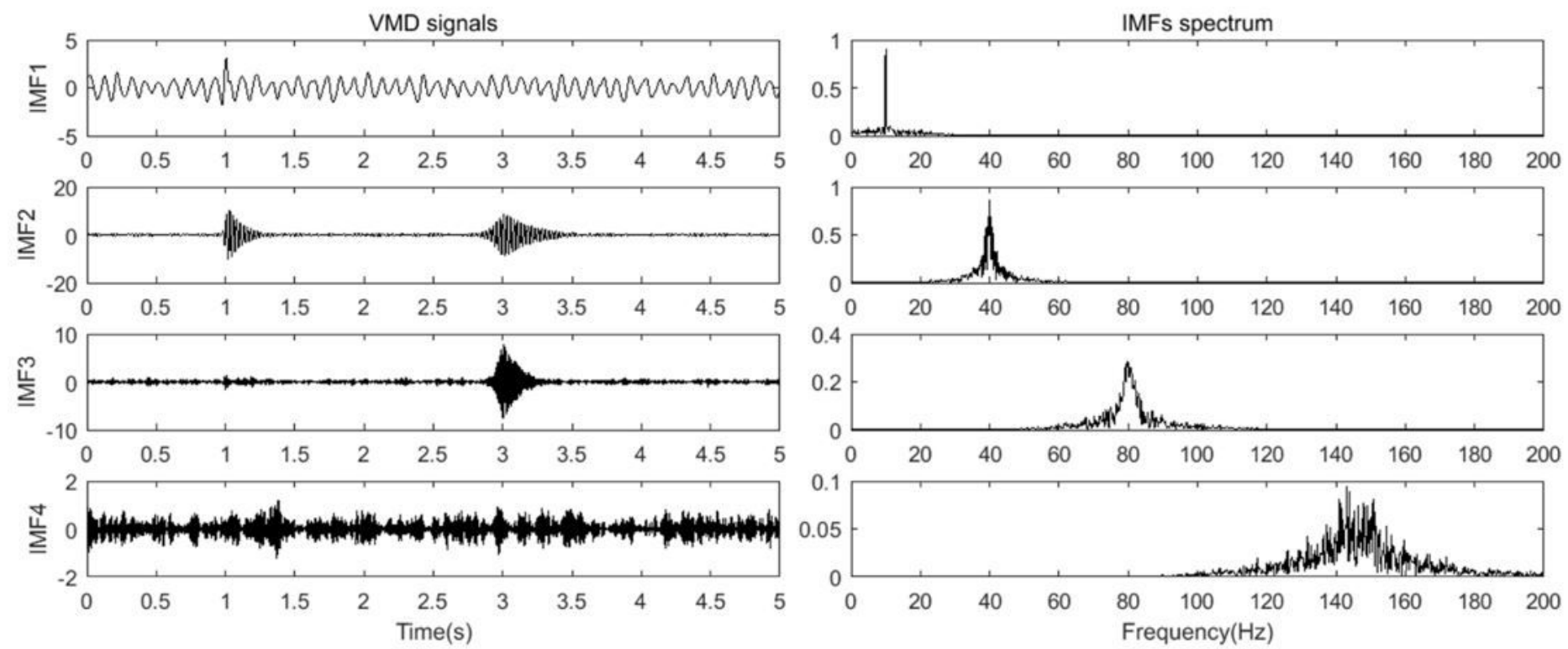

Figure 5

VMD decomposition mode signals and their frequency spectrums 

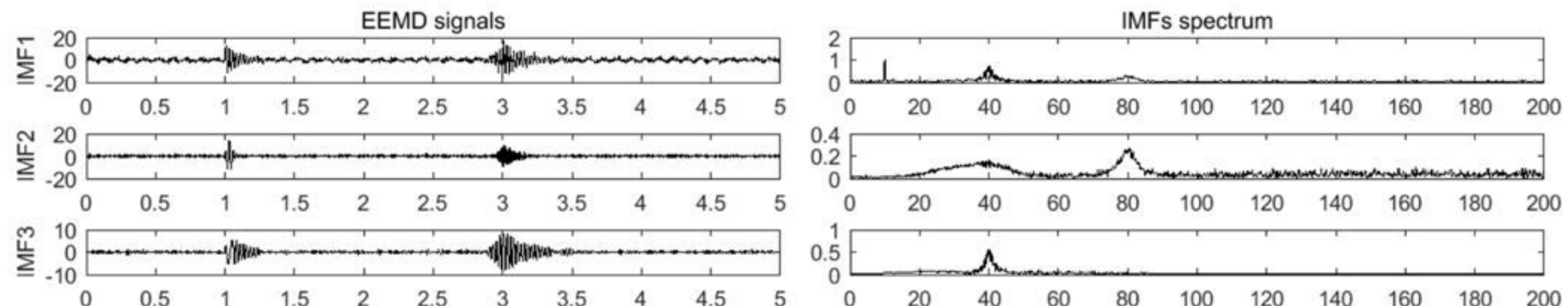

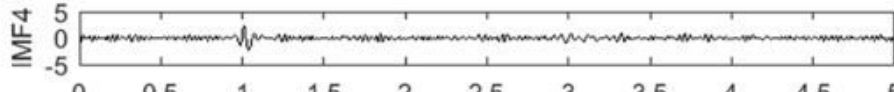

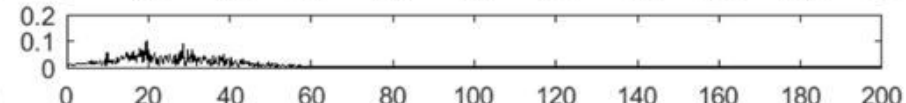

$\sum_{-2}^{2}$ Arron

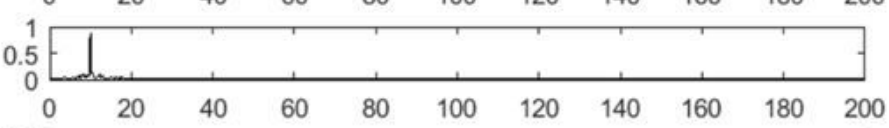

$\sum_{0.5}^{\infty} \sum_{1}^{1.5} \sum_{\substack{\text { Time(s) } \\ 2.5}}^{1}$

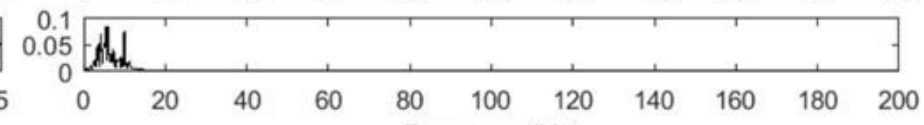

\section{Figure 6}

The simulation signal EEMD components and their spectrum

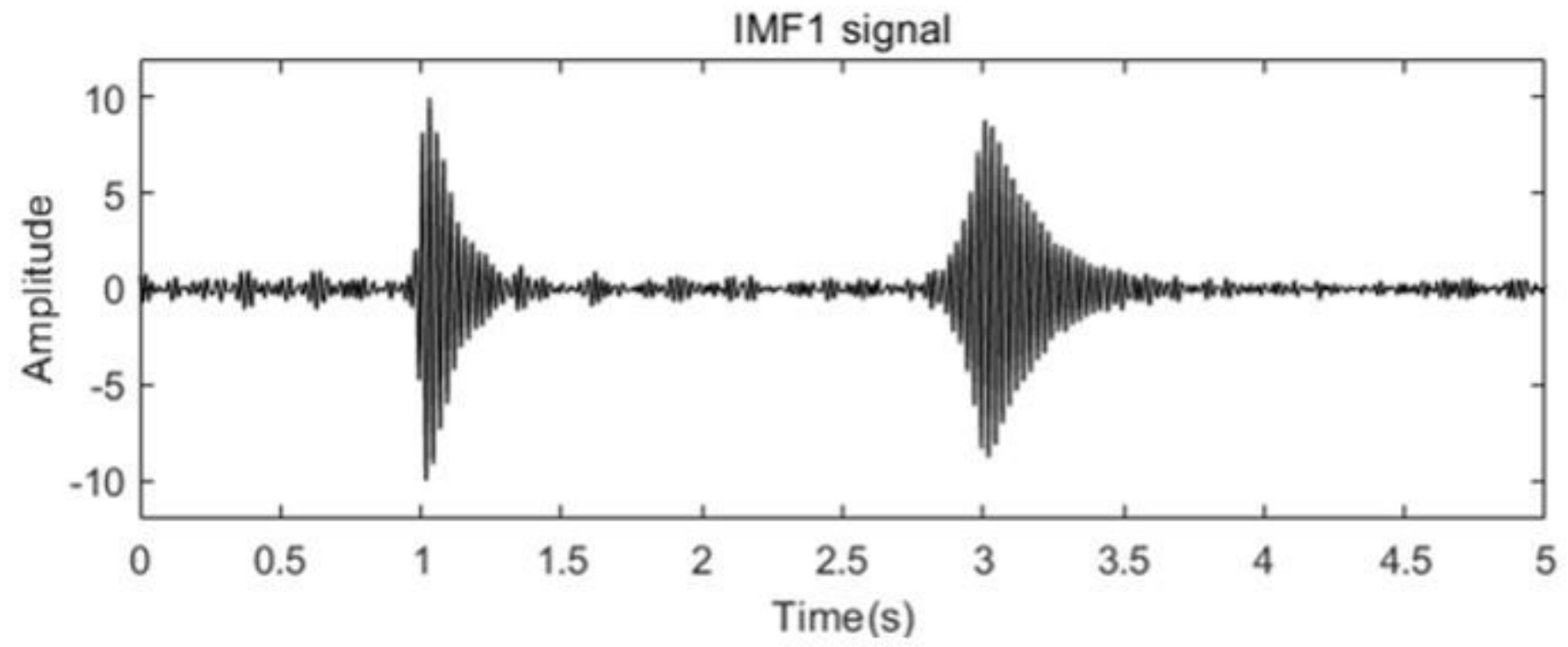

Figure 7

IMF1 signal 


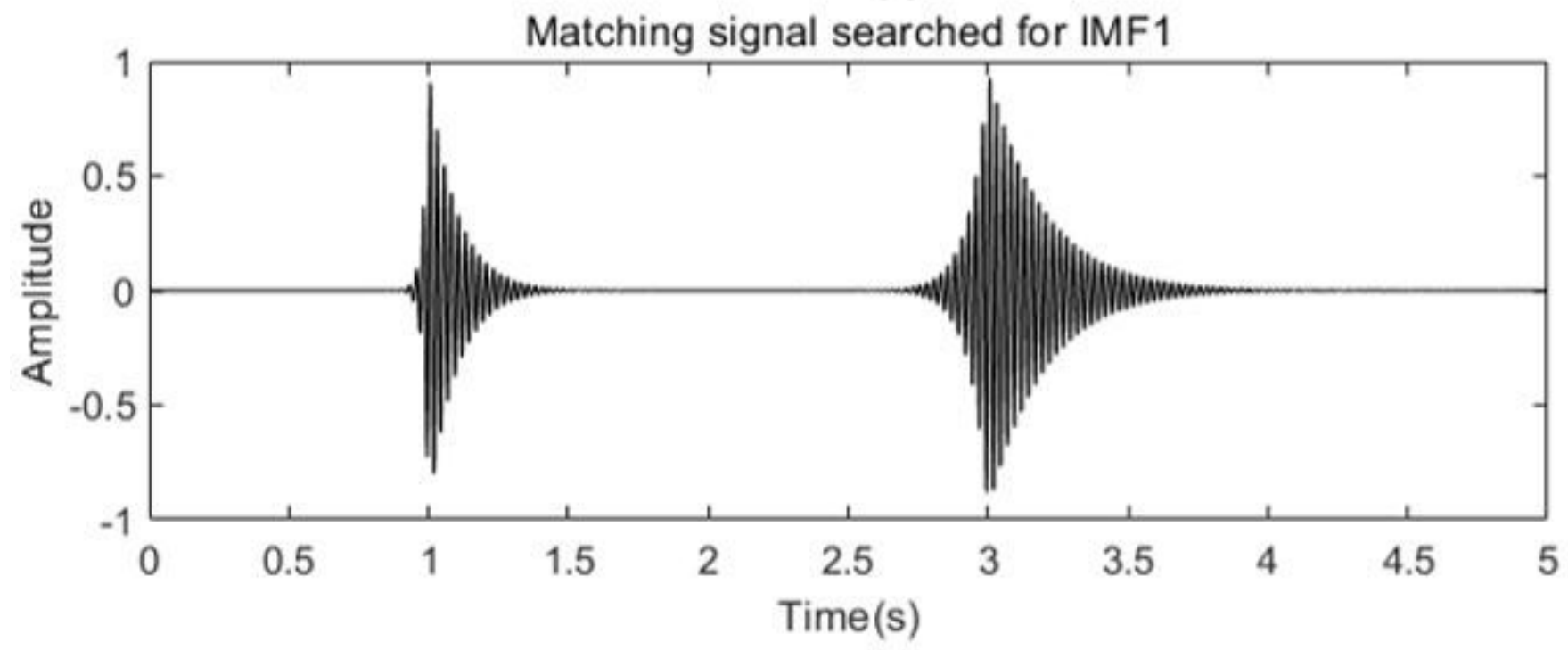

Figure 8

Signals constructed by parameters after searching

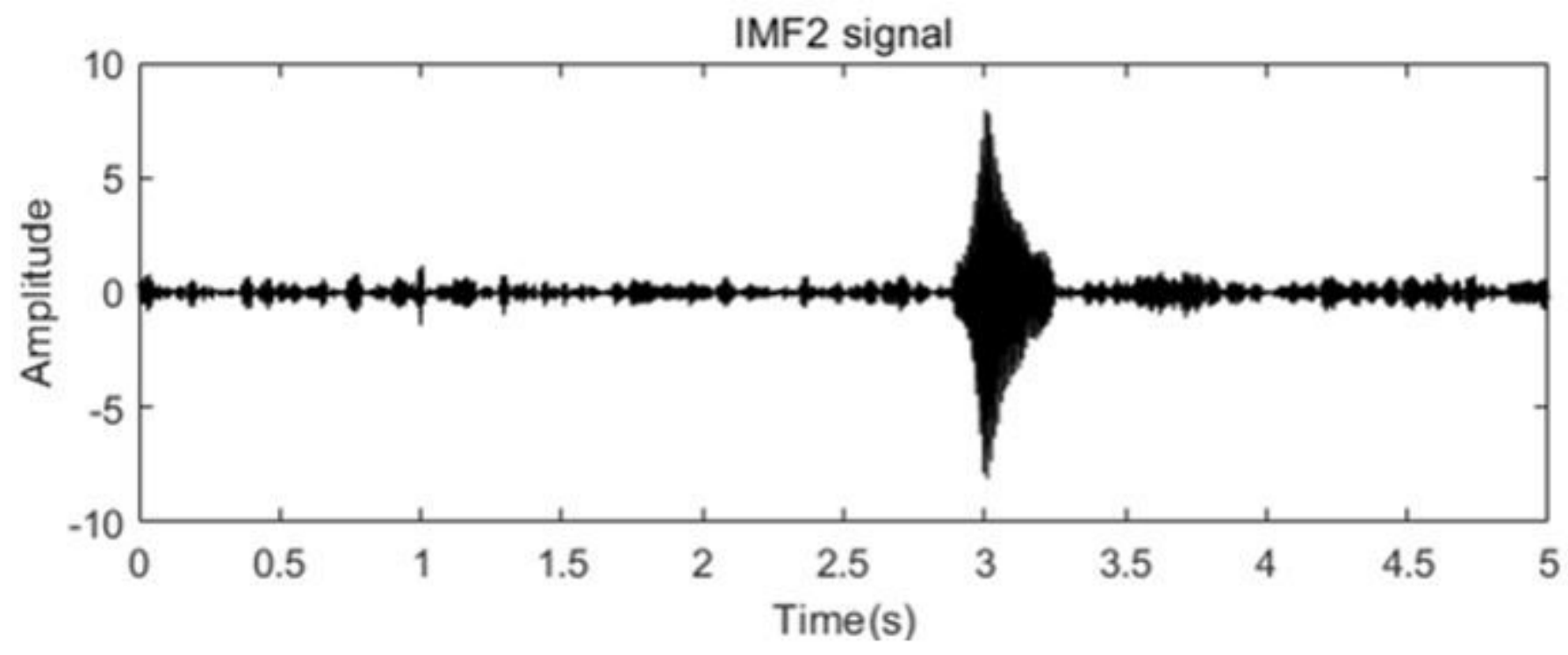

Figure 9

IMF2 signal 


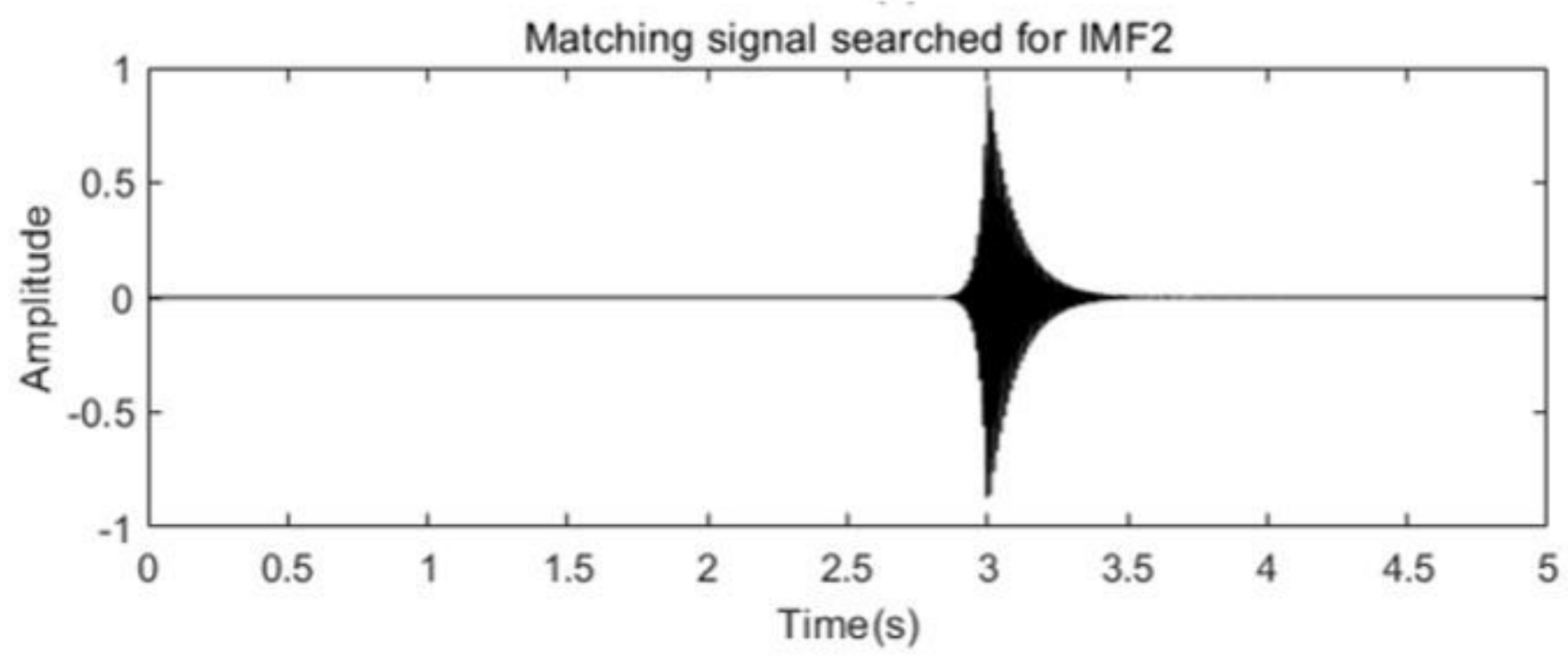

Figure 10

Signals constructed by parameters after searching

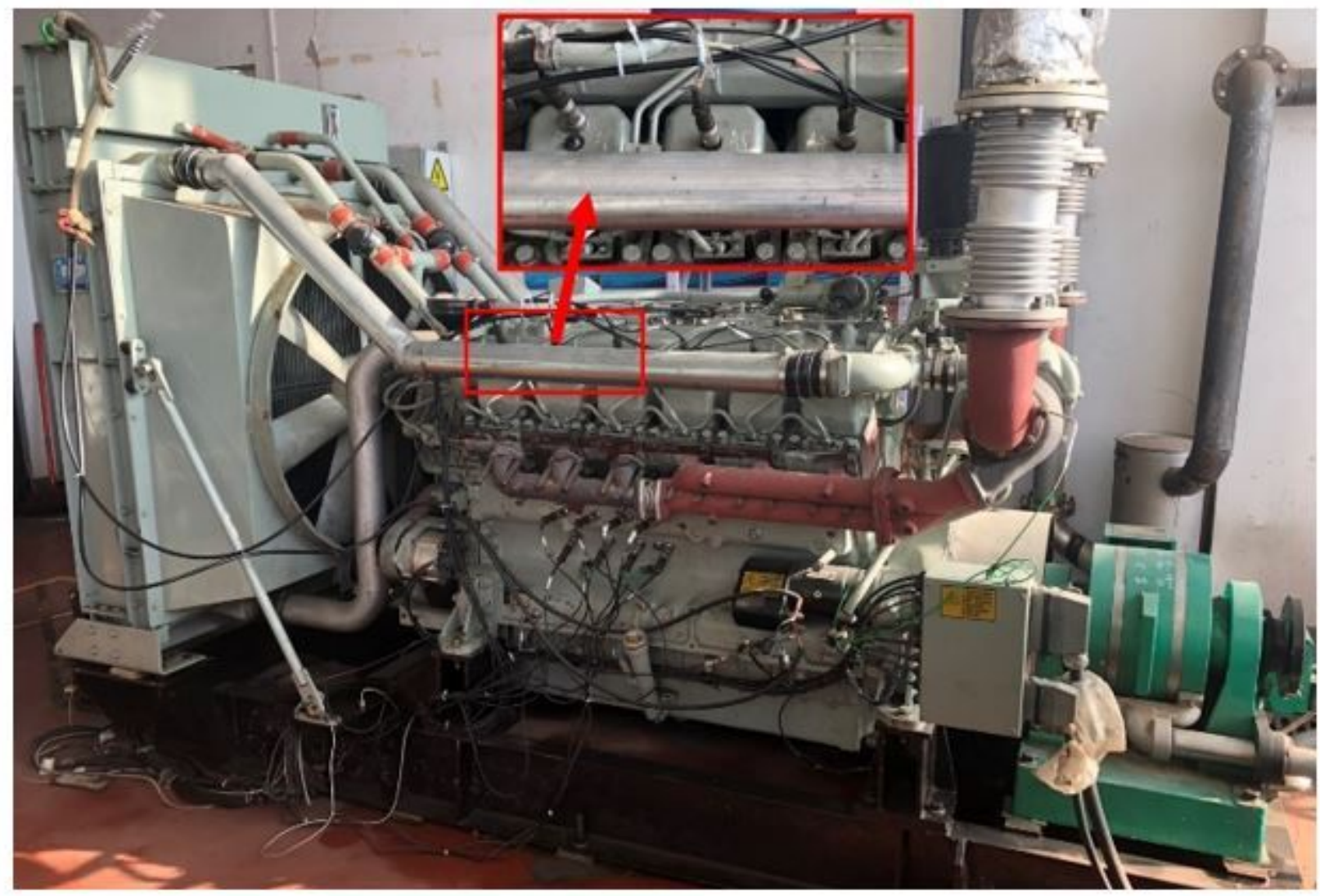

Figure 11 
Diesel engine and measuring point installation

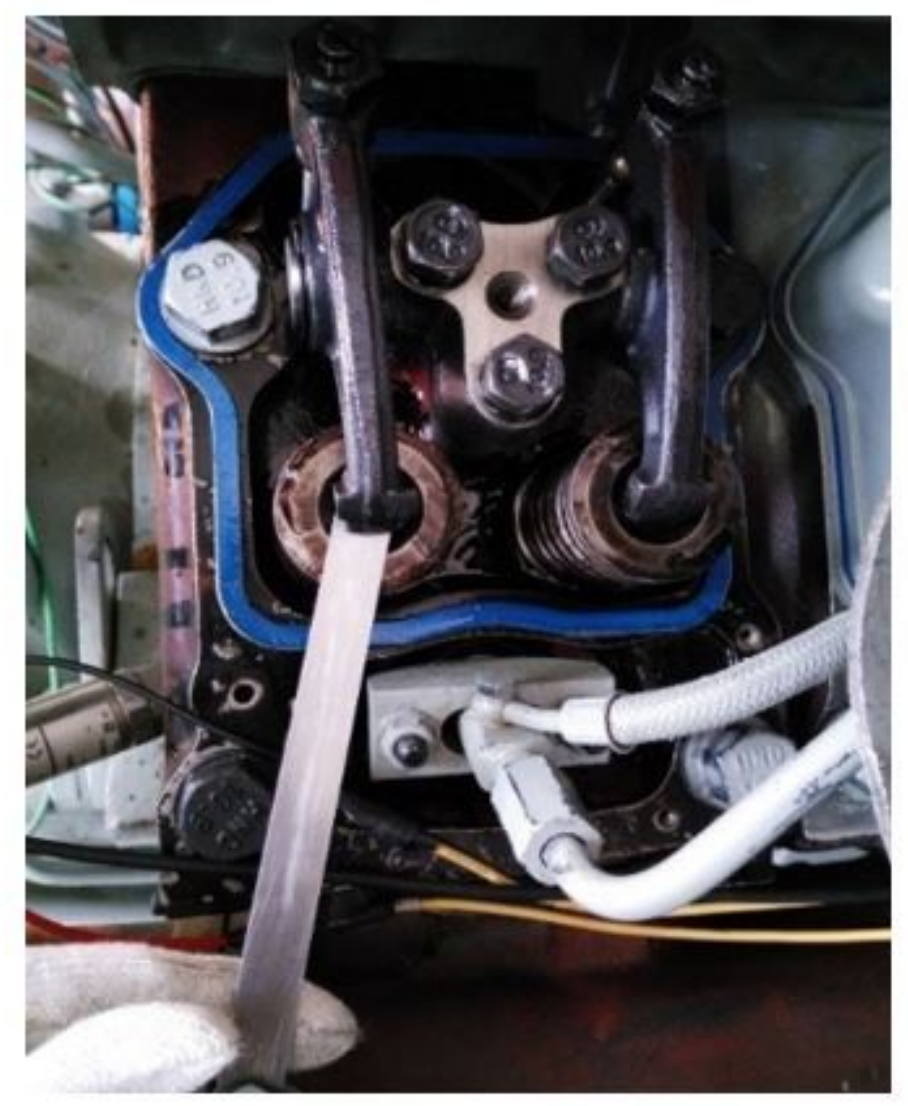

\section{Figure 12}

Valve clearance adjustment

B1 cylinder vibration signal

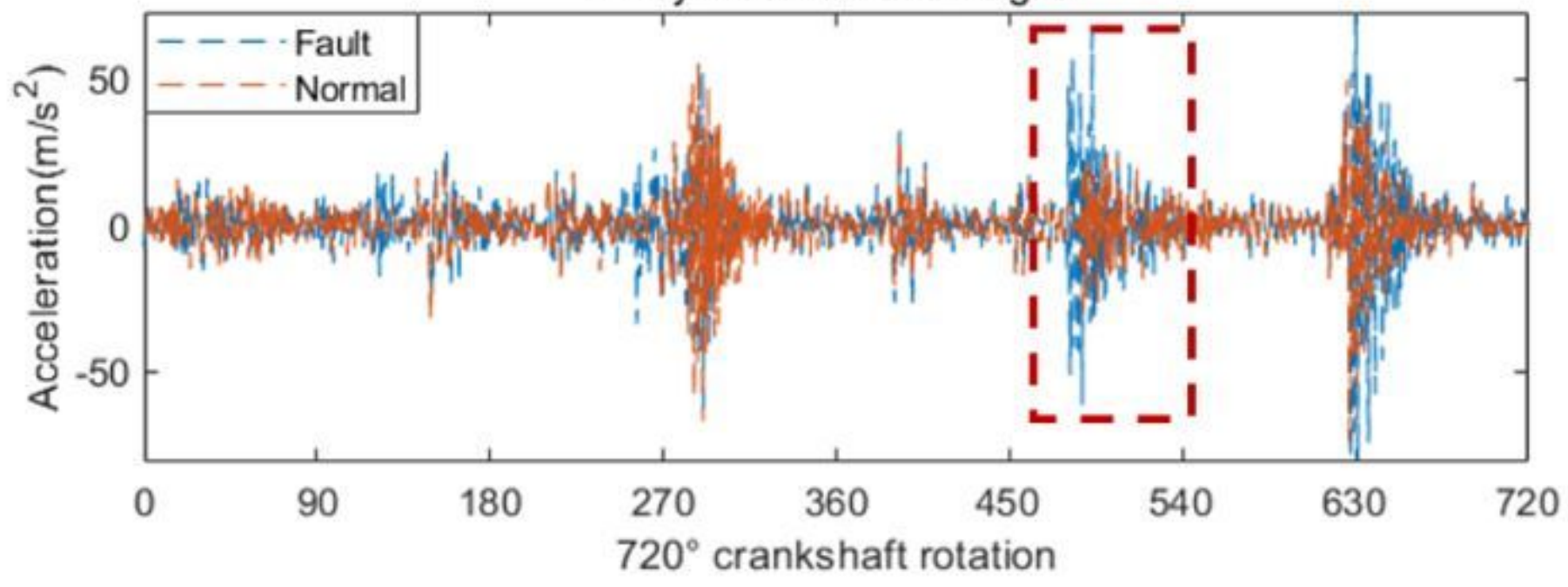

Figure 13 
Vibration signal of B4 cylinder under normal and fault conditions

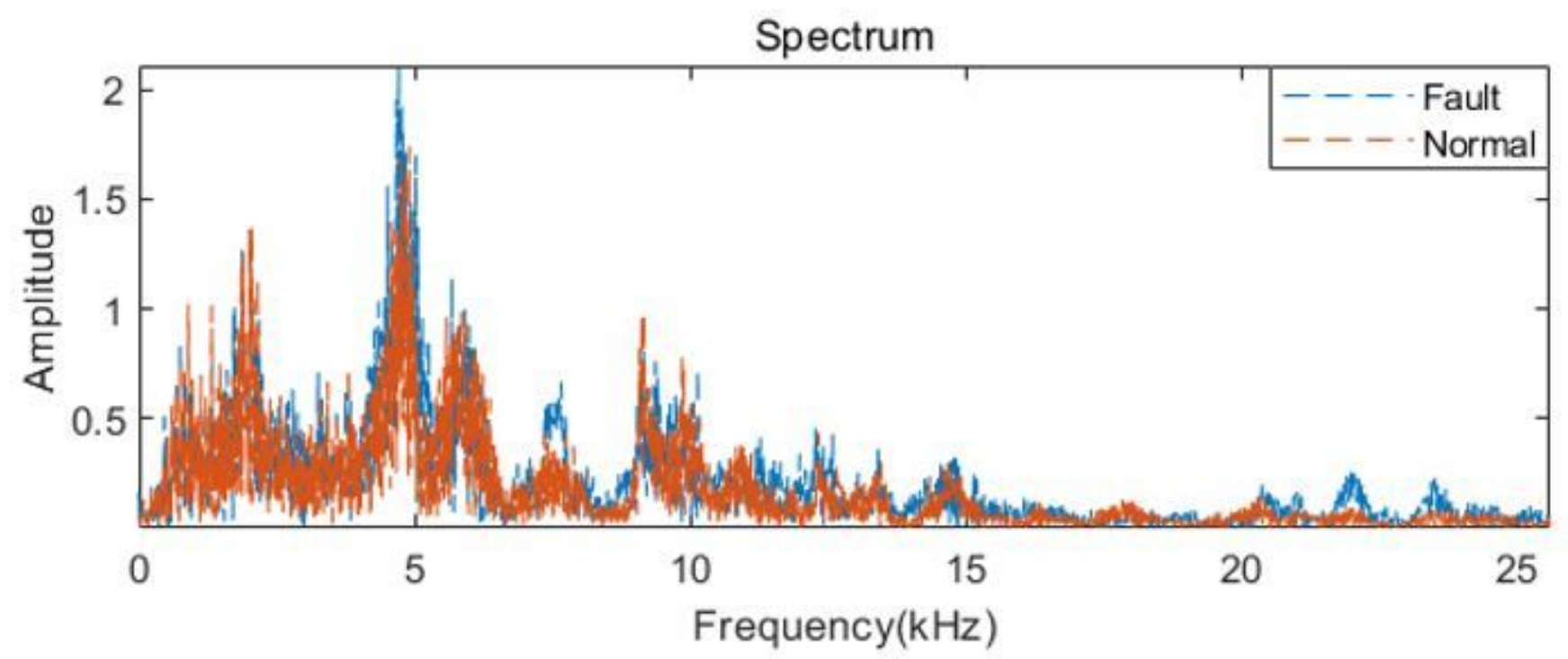

Figure 14

Vibration signal spectrum of B4 cylinder under normal and fault conditions

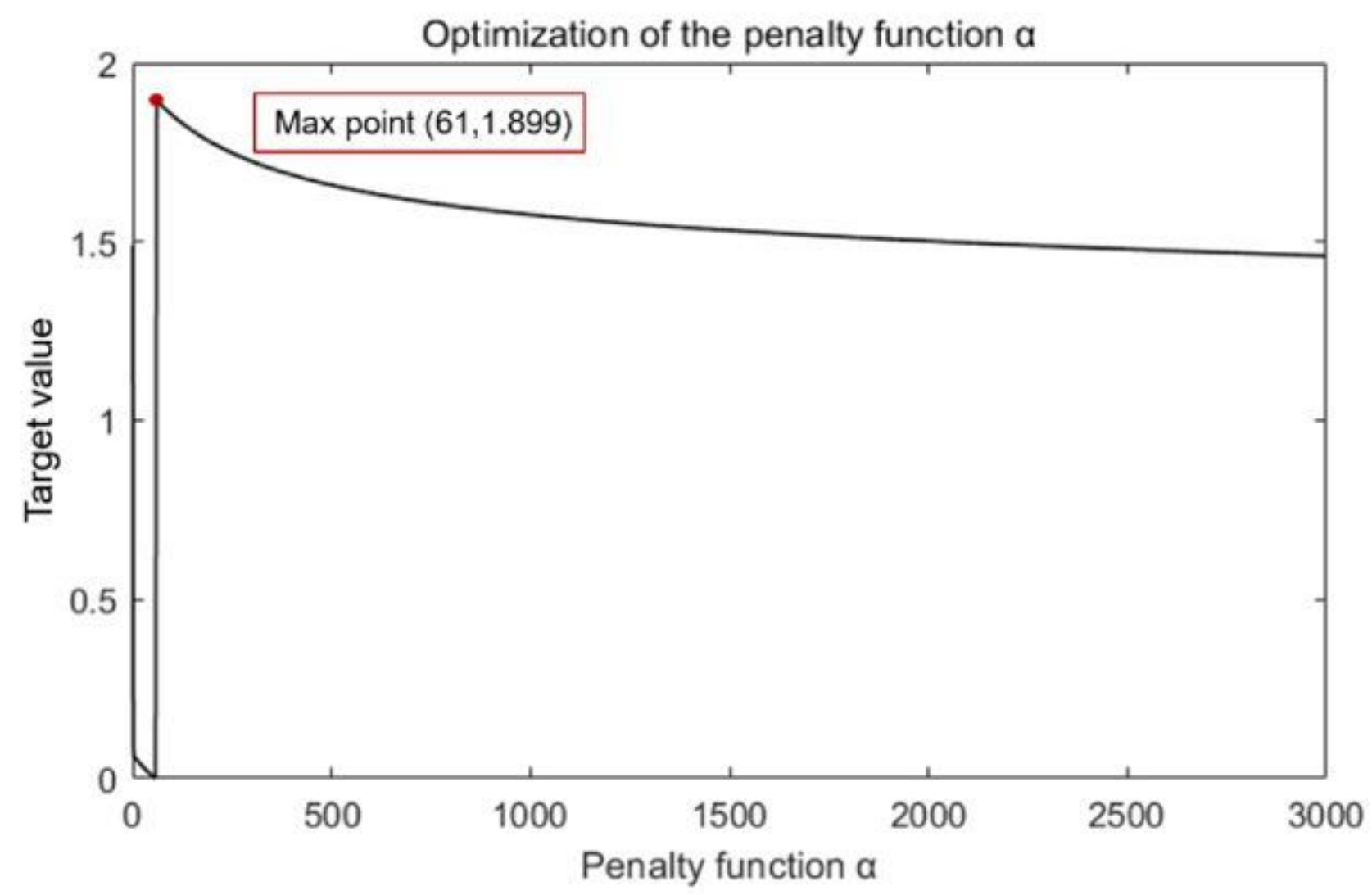

Figure 15 


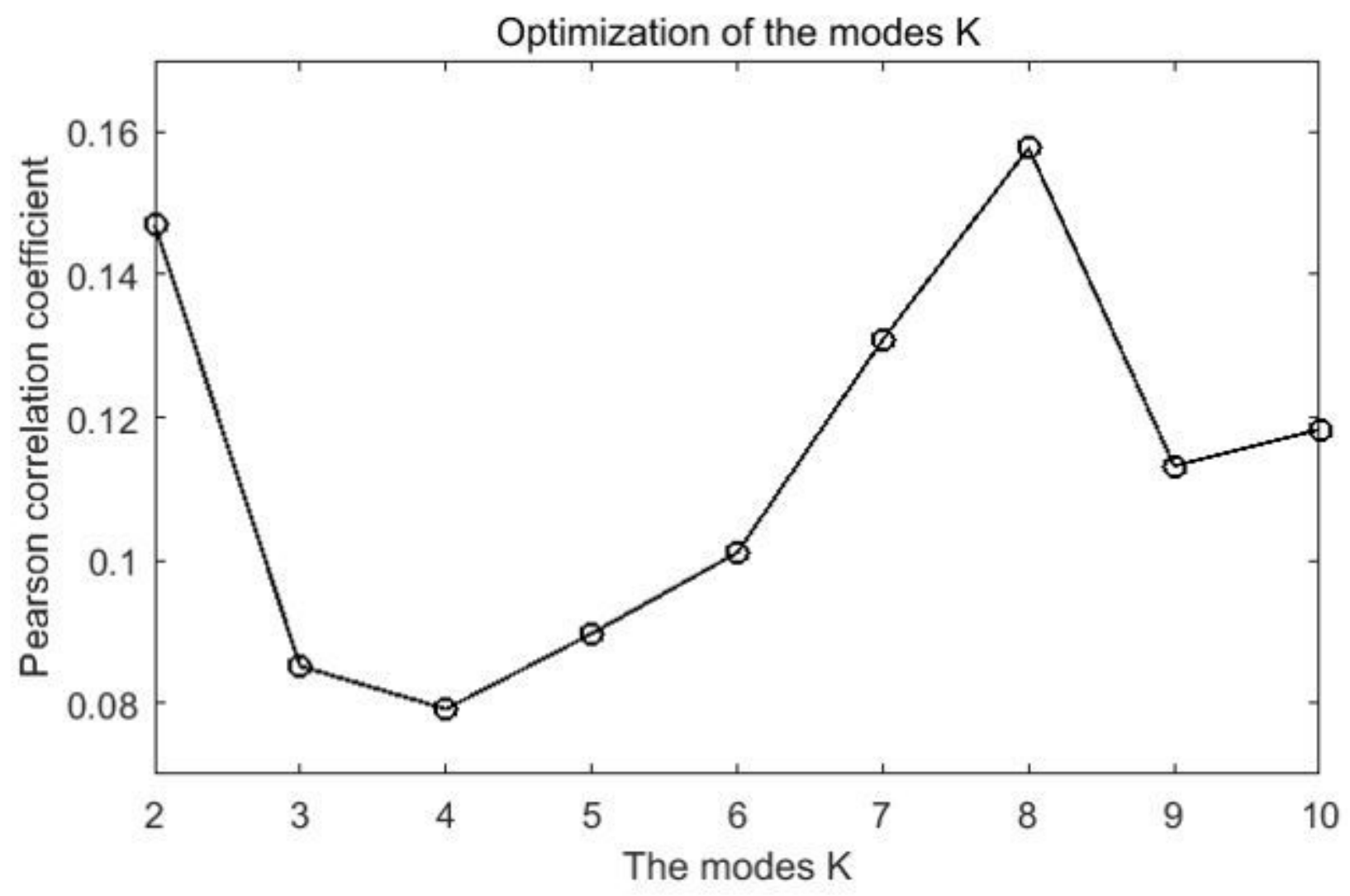

Figure 16

VMD decomposition iteration of the modes $\mathrm{K}$
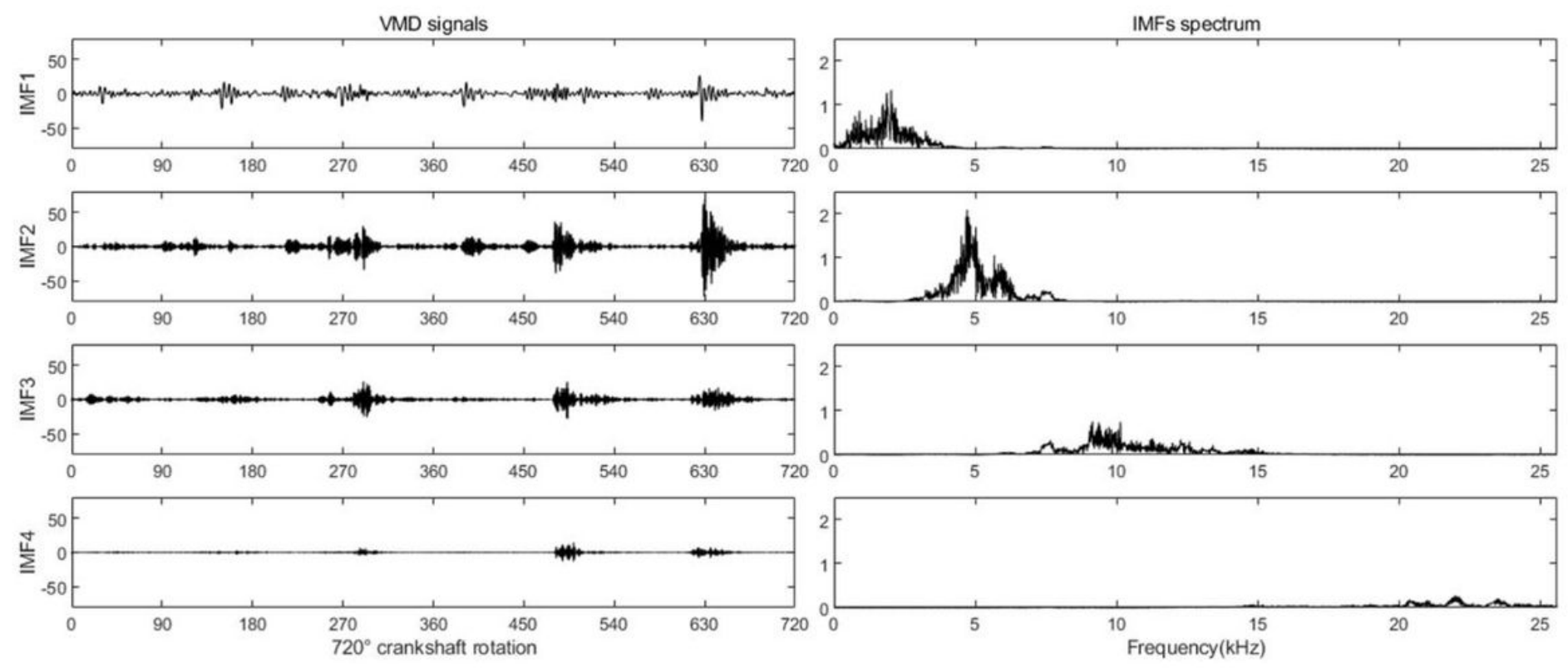

Figure 17 
VMD decomposition mode signals and their frequency spectrums

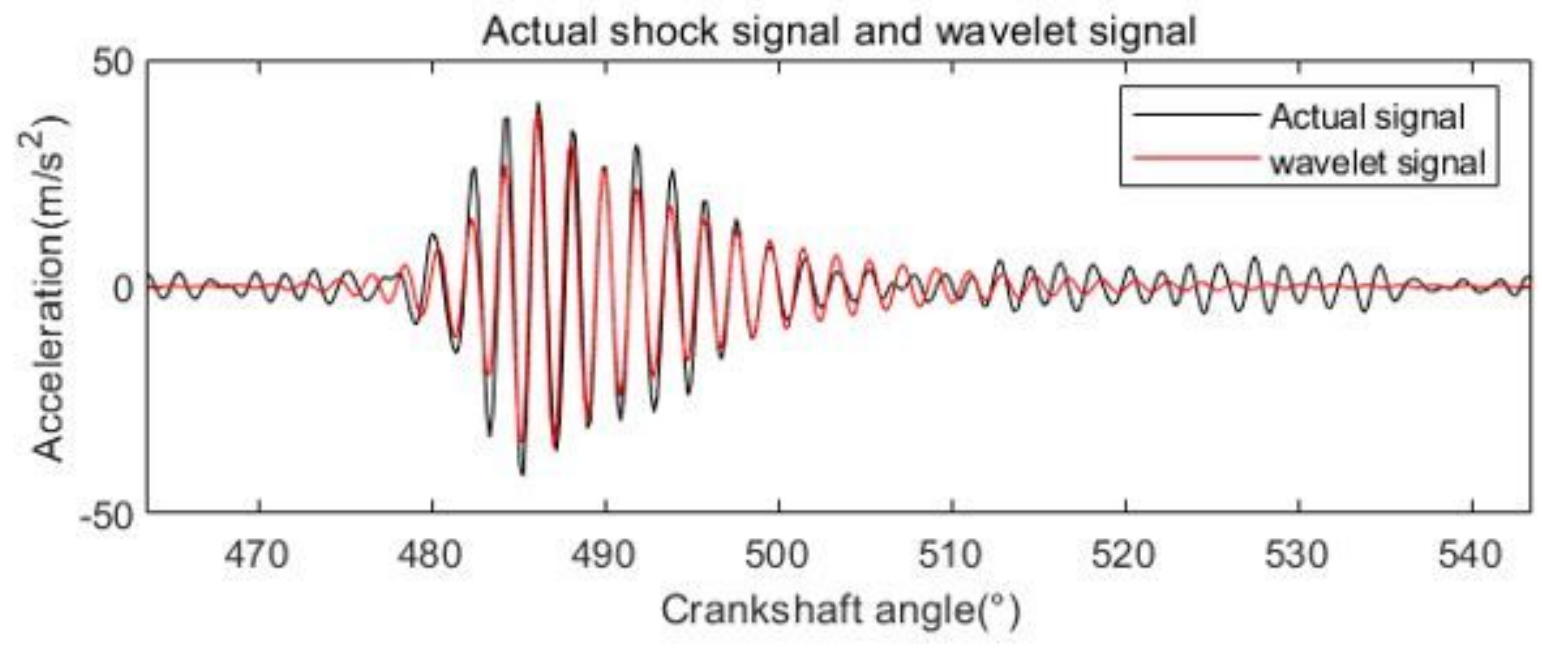

Figure 18

BALW search results

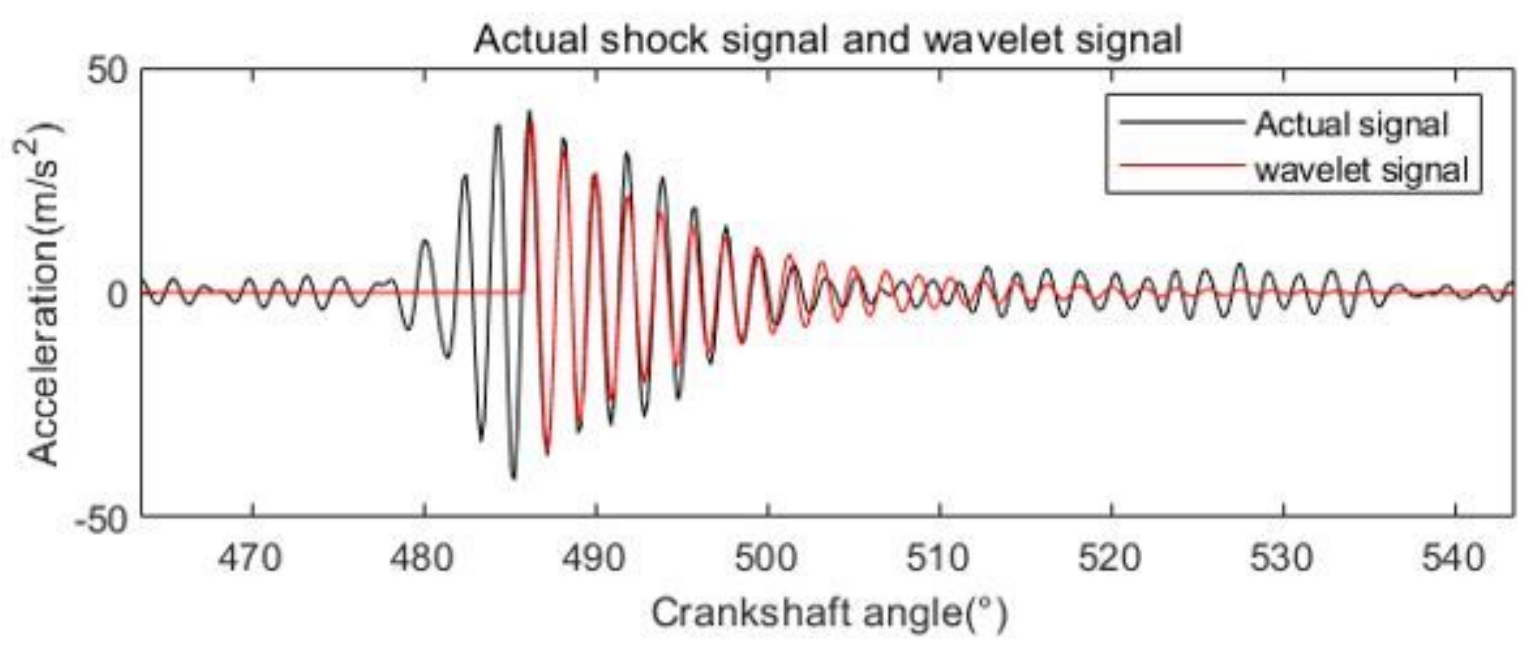

Figure 19

LW search results 
Actual shock signal and wavelet signal

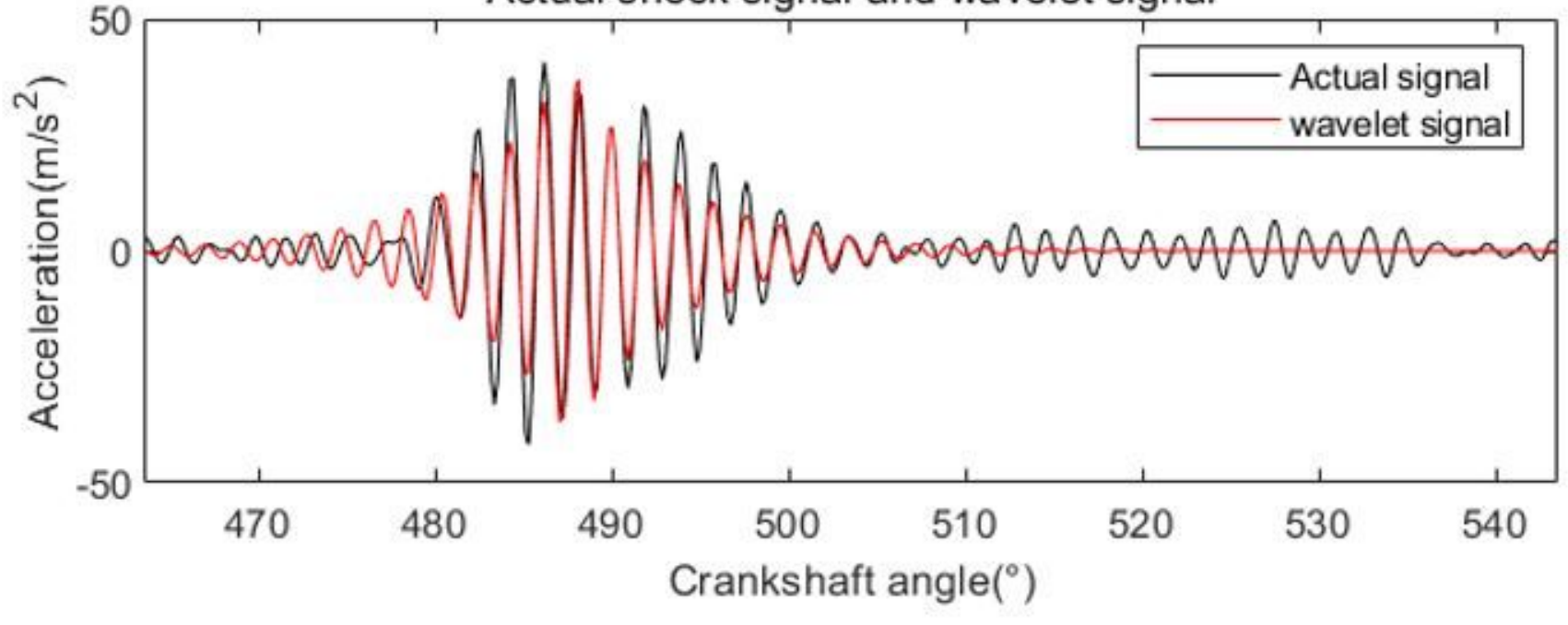

Figure 20

ARLW search results

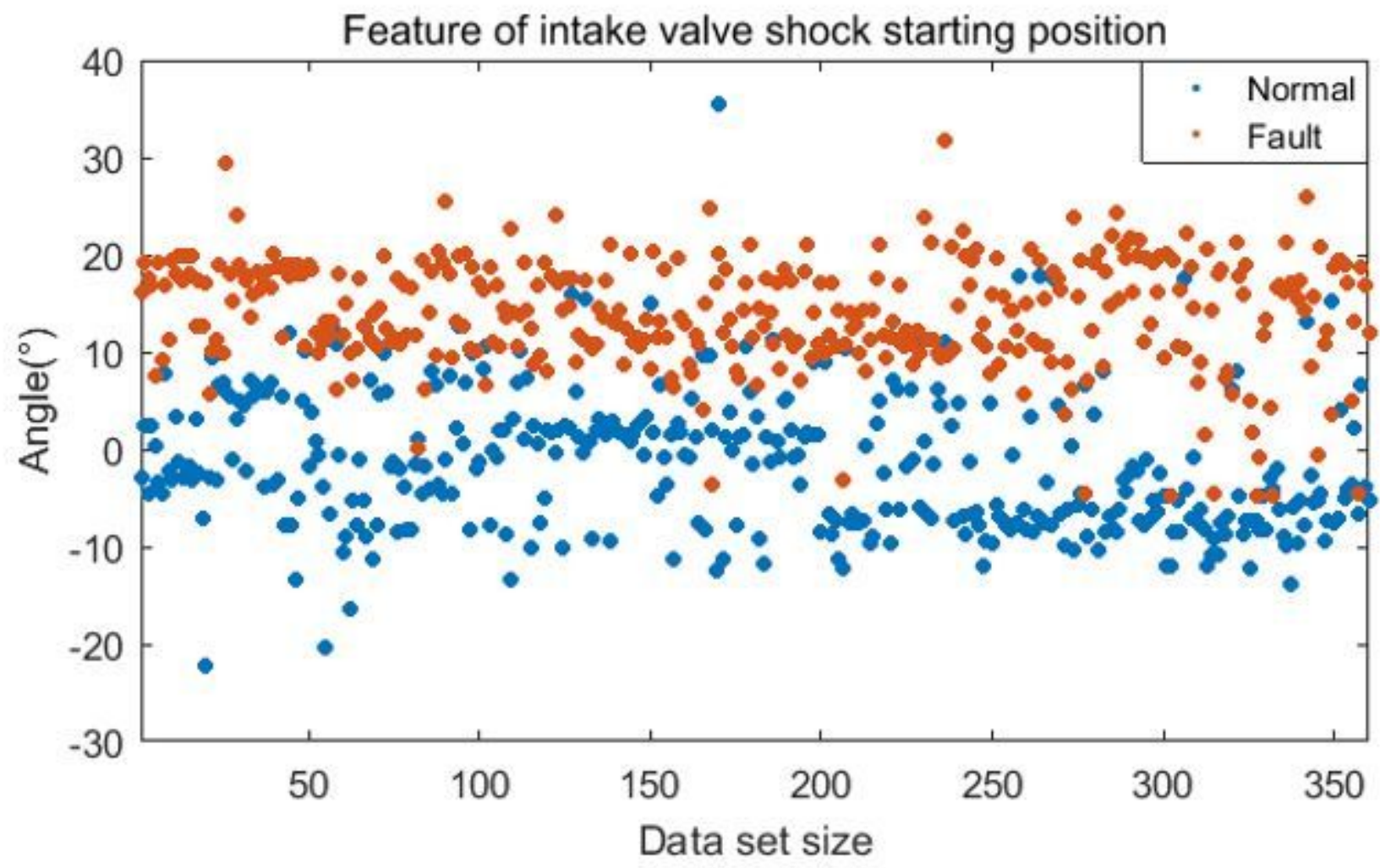

Figure 21

Characteristics of starting position of intake valve closing shock 
Feature of intake valve shock peak

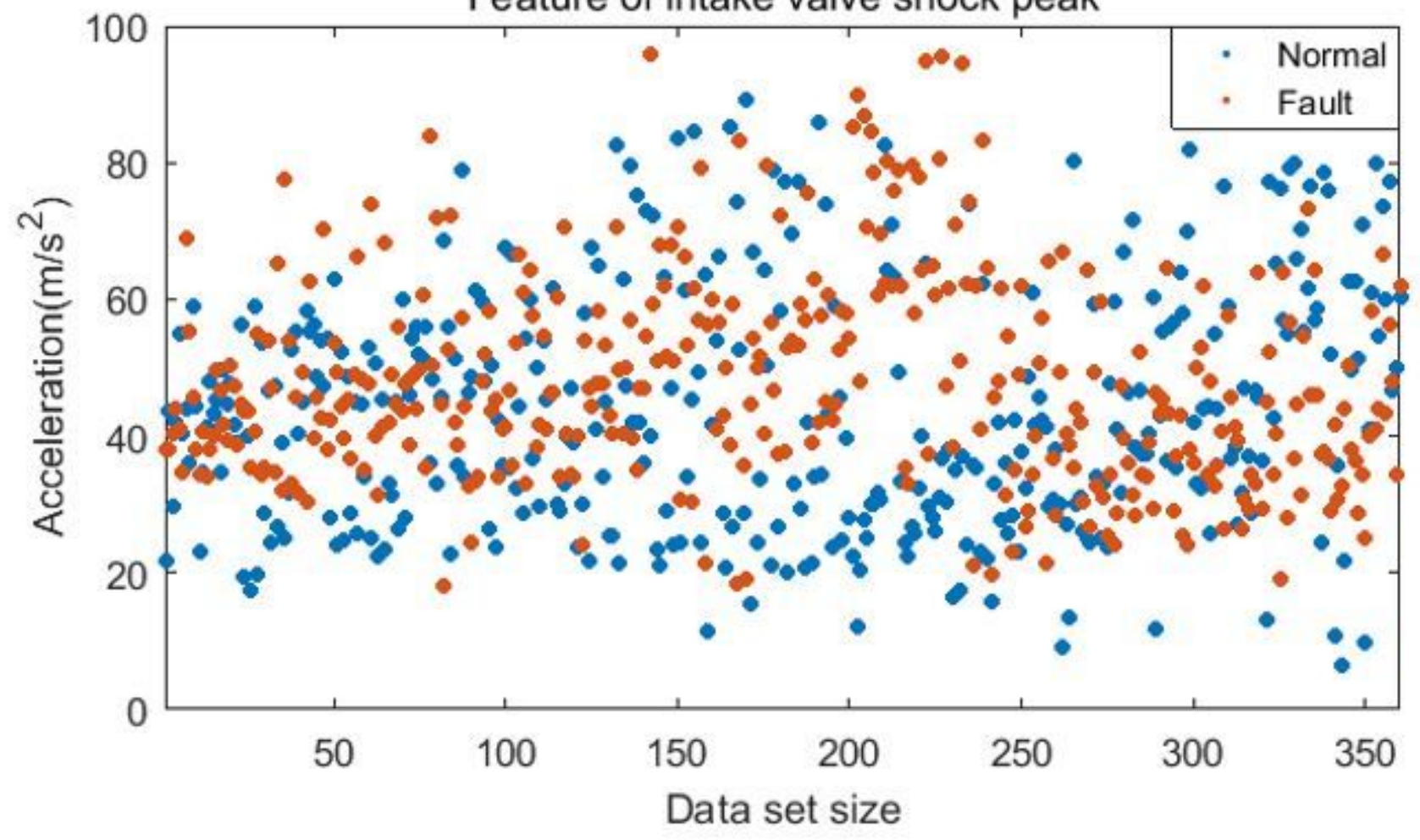

Figure 22

Figure 22 Characteristics of peak of intake valve closing shock 